\title{
Could Wading in Shallow Water Account for the Unique Shape of the Australopithecus afarensis Pelvis?
}

\author{
Algis Vincent Kuliukas \\ School of Anatomy, Physiology and Human Biology, University of Western Australia, Crawley, Australia \\ Email:algis.kuliukas@uwa.edu.au
}

How to cite this paper: Kuliukas, A. V. (2018). Could Wading in Shallow Water Account for the Unique Shape of the Australopithecus afarensis Pelvis? Advances in Anthropology, 8, 18-72.

https://doi.org/10.4236/aa.2018.81003

Received: November 16, 2017

Accepted: February 25, 2018

Published: February 28, 2018

Copyright $\odot 2018$ by author and Scientific Research Publishing Inc. This work is licensed under the Creative Commons Attribution International License (CC BY 4.0).

http://creativecommons.org/licenses/by/4.0/

Open Access

\begin{abstract}
A 3D Geometric Morphometric (GM) analysis of the shape of the pelvis and femur of various extinct hominids and extant humans and apes is described. Observed differences in shape are then discussed in the context of the wading hypothesis, a model of the evolution of hominin bipedalism that has rarely been seriously considered despite some compelling arguments in its favour. The general shape of the pelvis of Australopithecus afarensis is confirmed to be fundamentally different from both Homo and extant great apes, and not intermediate between them. Although it includes some human-like traits indicating a strong propensity to bipedalism, there are also sufficient differences to indicate that australopithecines probably exhibited a different type of bipedality to the relatively efficient striding gait associated with modern humans. An analysis of putative muscle lever arm ratios is described, which generated over 135,000 ratios in all. This data was then explored using the Pivot Table feature of Microsoft Excel. Succinct species summaries of broad lever arm groups, such as those pertaining to abduction compared to those pertaining to extension were generated. The results indicate that the australopithecine hip was more adapted, than modern humans or extant great apes, to adduction, abduction and rotation of the thigh during locomotion. It is argued that this apparent lateral biomechanical advantage complements the broad platypelloid shape as a putative adaptation to side-to-side wading. This adds further evidential weight to the wading hypothesis of bipedal origins in addition to the already compelling arguments from extant ape behaviour in shallow water and the favourable evidence of the paleohabitats of the earliest bipeds.
\end{abstract}

\section{Keywords}

Bipedalism, Australopithecine, Pelvic Shape, Wading Hypothesis, Bipedal 
Origins

\section{Introduction}

The shape of the australopithecine pelvis is now reasonably well known thanks largely to two major fossil finds (STS14, AL 288-1) which have been extensively studied (Le Gros Clark, 1955; Lovejoy \& Heiple,1970; Zuckerman et al., 1973; McHenry \& Corruccini, 1975; Stern Jr. \& Susman, 1983; Berge, 1984; Berge \& Kazmierczak, 1986; Häusler, 1992; Berge, 1994; Abitbol, 1995; Häusler \& Berger, 2001) some with the use of sophisticated geometric morphometric analysis (Oxnard, 1975; Ashton et al., 1981). Despite this work, there is still some dispute among anatomists about how to explain the peculiar, indeed unique, shape of the australopithecine pelvis and what it tells us about how they may have moved.

Although there is a broad consensus that australopithecines were generally bipedal in their locomotion, there remains disagreement about how human-like their form of bipedalism was. Some (e.g. Lovejoy et al., 1973; Lovejoy, 1979, 1981; Latimer, 1991; Crompton et al., 1998) have argued that the australopithecine post crania speaks not only of bipedality but a very human-like striding gait. Others (e.g. Stern \& Susman, 1983; Berge, 1984; Berge \& Kazmierczak, 1986) have argued that the evidence indicates a more ape-like bent-hip, bent-knee gait. Some (e.g. Oxnard, 1975, 1979; Ashton et al., 1981) even questioned if they were human ancestors. The former view seems to have gained in popularity in the last ten years or so, based on several research angles which appear to support, but are also based upon an assumption of, the human-like striding gait (Crompton et al., 1998; Sellers et al., 2004; Carey \& Crompton, 2005). However, it is argued here that several key pieces of evidence seem to have been overlooked in coming to such an interpretation.

Firstly, the very unusual, "platypelloid", shape of the pelvis has rarely been addressed. Unlike the general primate template, with its characteristically long (in the superior-inferior axis), narrow (in the lateral dimension) hip, australopithecines are almost at the opposite end of the spectrum, being very short (in the superior-inferior axis) and relatively wide, laterally. As a species that appears to have been somewhat arboreal, as evidenced by its curved phalanges, and somewhat bipedal, as evidenced by the large bicondylar angle of the femur, one might expect its shape to be somewhat intermediate between the shape of great apes and humans. Instead, its relatively flat and wide shape is a peculiarly unique outlier to the whole Primate Order.

Secondly, the rather wet and swampy palaeohabitat of the Hadar, the site of AL-288-1 and others also seem to have been overlooked. Aquatic fauna, found alongside "Lucy", indicated that moving through water was a plausible occasional mode of locomotion for these hominins. However wading in shallow water is 
conspicuous by its absence from the literature as a putative mode of bipedalism in australopithecines and, instead, the assumption has been that their bipedality must have been on dry land, in somewhat open, savannah-like, habitats.

\section{The "Obstetric Dilemma"}

Much that has been published on the shape of "Lucy's" pelvis has been focused on child bearing aspects which have been predicated on the assumption that Lucy was female, (although see Häusler \& Schmid, 1995). The so-called "obstetric dilemma" has been a topic of discussion for decades of students and the assumption that the shape of the modern human female pelvis is something of a compromise from the male shape, which is more optimised for bipedality, is well established. The need to give birth to large-headed infants has required this evolutionary compromise for wider female pelves, or so the argument goes.

A recent reassessment of fossilised pelvic fragments of Sts 65 concurred with the already largely accepted platypelloid shape of AL 288-1, and also a likely female gender of the sample (Claxton et al., 2016). By careful reconstruction of the pelvis and comparison with known australopithecine cranial size, they concluded that a human-like oblique or transverse entry of the infant head during labour would have been well established even by 3.2 Ma.

However, this putative correlation between pelvic shape and obstetrics does not demonstrate causality. That the australopithecine pelvis was so platypelloid as to require an oblique or transverse entry for the fetal head into the pelvis is not in accordance with the reason attributed to the human condition, which is to allow the birth a large infant head. The size of the australopithecine fetal head seems likely to have been similar to that of chimpanzees (Kimbel et al., 1994). The obstetric dilemma argues that the slightly more platypelloid human female pelvic shape is a compromise from the, presumably, more energetically efficient, narrower, male form to aid child birth. But in this regard the australopithecine pelvis seems to indicate marked energy inefficiency without any compensatory obstetric advantage.

Therefore, the shape of their pelvis marks an outlying position, among the whole primate order, long before the process of encephalisation could have caused the possibility of cephalopelvic disproportion which would then drive the evolutionary response we see in the female pelvis today. Here is a pelvis, indicative of bipedality, that was even wider than the most platypelloid of all modern female pelves-but clearly not driven in any way by an adaptive shift to help in child birth.

It begs the question: Could something else have been driving the platypelloid shape?

\section{Fully Upright v Bent-Hip Bent-Knee, Revisited}

In the 1990s, the paleoanthropological literature regularly featured papers discussing how australopithecines moved. Their bipedality was generally accepted 
but how they walked was disputed. Some, e.g. Lovejoy (1979), suggested that their anatomy was consistent with human-like fully-upright bipedalism, whilst others (e.g. Stern Jr. \& Susman, 1983) disagreed, suggesting that it was more indicative of an ape-like bent hip, bent knee gait.

The evidence given by both sides was compelling. For example, the bicondylar angle of the distal femur in australopithecines seems to be consistent with what one might expect if their anatomy was, like modern humans, optimised for an efficient fully-upright, human-like, striding gait, where the knee is placed under the center of mass during the stance phase.

Equally, however, the shape of the australopithecine pelvis seemed to be inconsistent with this, suggesting a waddling, bent-hip bent-knee gait to many experts (e.g. Berge, 1994). Several features, such as curved phalanges, indicate a degree of arboreality which suggested that they were perhaps not yet obligate bipeds.

Some studies of the australopithecine pelvis (e.g. Zuckerman et al., 1973) set out to objectively analyse the shape mathematically. When Oxnard \& Wilkes later summarised over two decades of such studies, they wrote:

"The deduction that stems from the investigations just described is that, because the form of the pelvis in the fossil is neither human-like nor ape-like but uniquely different from both humans and apes, the fossil, therefore, must have had a form of locomotion uniquely different from that of both apes and humans" (Oxnard \& Hoyland-Wilkes, 1994: p 19).

They added...

"Not being willing to believe that this actually meant a totally unique form of locomotion (such as jumping up and down on its thumbs), we accepted that a unique combination of several activities (perhaps the combination of terrestrial bipedal stance and locomotion with arboreal quadrupedal running, climbing and aerobatics) might require a pelvis of unique structure." (Oxnard \& Hoyland-Wilkes, 1994: p 19)

However it occurred to this author that such a combination of postures and forms of locomotion currently exhibited in extant primates from time to time, would be expected to lead to a form of pelvis that was a combination or compromise of such extant pelves, not generate a completely novel form. It seemed to me that perhaps all that was missing in Oxnard and Hoyland-Wilkes' analysis was to propose a mode of bipedal locomotion that was plausible in ape-like ancestors, but that was sufficiently different from human walking to have selected for a quite different anatomical shape. Could this also be a form of bipedalism that might have generated a set of seemingly contradictory traits like those just mentioned, and yet somehow explain them all?

\section{The Wading Hypothesis}

There is a scenario, rarely considered in the paleoanthropological literature, that can very predictably induce sustained, unsupported, bipedal locomotion in ex- 
tant great apes. I stress the term sustained, as many models of hominin bipedal origins can merely point to isolated, transitory, instances of upright posture that rarely last more than a few seconds. In waist deep water, great apes are compelled to switch from quadrupedalism to bipedalism, without the use of their upper limbs for support. They are compelled to do so potentially indefinitely, certainly for as long as the conditions prevail. Therefore, there seems to be no better candidate than wading in shallow water to answer the dilemma posed by Oxnard et al. above.

Although first articulated by Westenhöfer (1942) and independently by Hardy (1960) several decades ago, the idea that wading through shallow water may have been a key factor in the evolution of human bipedality has not received much broad attention from the field of anthropology, despite a great deal of individual work by Morgan (1972, 1982, 1990, 1993, 1997) and others (e.g. Ellis 1993; Niemitz, 2002, 2004, 2007, 2010; Kuliukas, 2002, 2011, 2013, 2017; Kuliukas et al., 2009; Verhaegen et al., 2002). Wading also happens to be a putative mode of locomotion entirely consistent with the known palaeohabitat of Hadar (apparently, a wetland for over a million years Johanson \& Edey, 1981: p. 128) and the habitats of other early hominin bipeds (e.g Sahelanthropus, see Vignaud et al., 2002).

Could a wading gait be consistent with the australopithecine's rather large bicondylar angle and also explain its peculiar pelvic shape? This paper considers these possibilities in the light of a 3D GM study designed to compare the overall shape of the australopithecine hip to humans and extant apes and then to attempt to draw out evidence pertaining to the possibility of wading in the australopithecine locomotor repertoire by analysing Lever Arm ratios of putative hip muscle formations.

It should be conceded that since I came across Charles Oxnard's and his collaborators' pioneering work and started my own morphometric investigations into the shape of the australopithecine pelvis, a growing consensus seems to have gathered around the position that they exhibited a modern human striding kind of gait and those who argued against this in the 1990s have been largely silent.

The conclusions of this paper will attempt to redress the balance and provide good evidence that australopithecines did not walk like us at all but exhibited a rather unique form of bipedal locomotion that has not really been seen since.

\section{The "Wading Hypothesis" Compared to Other Models of Hominin Bipedal Origins}

Depending on one's method of classification, there are perhaps more than 30 unique published models on the origin of hominin bipedalism. A survey of university-level text books about human evolution found three broad ideas were the most commonly and favourably discussed: Carrying, Energy efficiency and postural feeding (Kuliukas, 2017).

One of the least popular models, that is rarely mentioned let alone impartially 
evaluated, is the wading hypothesis:

The wading hypothesis of hominin bipedalism argues that wading in shallow water (waist deep) was a key behaviour of the earliest hominin bipeds.

If one objectively tries to evaluate the relative strengths and weaknesses of the various published hypotheses (See later and

http://www.tinyurl.com/BipedalModels for a detailed and open account of the assessments of this author), one should first come up with a set of characteristics that might be expected of the better ones. For example, one would expect that the fossil evidence of the earliest putative bipedal hominids would be consistent with the hypothesis. Both the paleohabitats and the skeletal remains should support, and not contradict, the proposed behavioural scenario. Put in this context, the peculiarly platypelloid shape of the australopithecine pelvis poses a challenge to published models of bipedalism. How does the energy efficiency argument account for Lucy's hips? What about the carrying model or postural feeding?

The purpose of this study was to investigate the possibility that the platypelloid pelvis is consistent with the wading hypothesis.

Testable hypotheses...

Two null hypotheses are formulated here.

1) There is no significant difference between the shape of the australopithecine pelvis and that of modern human.

2) If there is a difference, it is not consistent with what one might predict if the difference was accounted for by more wading.

The next two sections of this paper will test these hypotheses.

\section{General 3D GM Study}

\subsection{Introduction}

Following on from McHenry \& Corruccini (1975) but using modern 3D geometric-morphometric techniques (see, e.g. Franklin et al., 2005 for other examples), a thorough analysis of the shape of the pelvis was conducted in great apes to determine the relationship between shape of the australopithecine pelvis and extant apes and modern humans.

See Supplementary Materials for details of the methods used.

\subsection{Results}

\subsubsection{General 3D Morphological Study}

A morph set comprising 125 hip bones from 23 species (see Table 1) was chosen for the study of the pelvis. A slightly smaller set of 102 Specimen contributed to the study of the sacrum. 71 femora specimen were used.

For those species with ten or more samples collected, females are represented with a smaller rectangle or circle.

In the Principal Component Figures 1-4 that follow the wire diagrams represent the shape of the morph at the position of the cloud indicated. 
Table 1. Species of pelves analysed.

\begin{tabular}{|c|c|c|c|c|}
\hline \multicolumn{5}{|c|}{ Species Studied } \\
\hline Phylogenetic Grouping & Latin Name & Code & $\mathbf{N}$ & Symbol \\
\hline 1 & Lemur catta & LEC & 1 & $\diamond$ \\
\hline 2 & Ateles geoffroyi & ATG & 1 & $\diamond$ \\
\hline 3.01.1.1 & Cercopithecus aethiops & CEA & 2 & $\bullet$ \\
\hline 3.01 .2 & Mandrillus sphinx & MSL & 1 & $\bullet$ \\
\hline 3.01 .3 & Theropithecus gelada & TGL & 1 & $\diamond$ \\
\hline 3.01 .4 & Nasalis larvatus & NSL & 2 & $\diamond$ \\
\hline 3.02 .1 & Hylobates syndactylus & HYL & 4 & $\Delta$ \\
\hline 3.02 .2 & Hylobates agilis & HYL & 2 & $\triangle$ \\
\hline 3.02 .3 & Hylobates lar & HYL & 1 & $\triangle$ \\
\hline 3.02 .4 & Hylobates moloch & HYL & 5 & $\triangle$ \\
\hline 3.02 .5 & Hylobates muelleri & HYL & 3 & $\triangle$ \\
\hline 3.03 .1 & Pongo abelii & PPA & 5 & 붑 \\
\hline 3.03 .2 & Pongo pygmaeus & PPY & 11 & 눕 \\
\hline 3.04 .1 & Gorilla gorillagorilla & GGO & 17 & E \\
\hline 3.04 .2 & Gorilla gorilla graueri & GGB & 10 & 뭄 \\
\hline 3.05 & Pan paniscus & PNP & 19 & $\mathbf{\square}$ \\
\hline 3.06 & Pan troglodytes & PNT & 16 & $\overline{\mathbf{n}}$ \\
\hline $3.07 \mathrm{X}$ & Australopithecus afarensis (AL 288-1) & AFA & 1 & 米 \\
\hline $3.09 \mathrm{X}$ & Homo erectus $(W T 15000)$ & HER & 1 & $x$ \\
\hline 3.10 & Homo sapiens sapiens & HSS & 32 & - \\
\hline
\end{tabular}

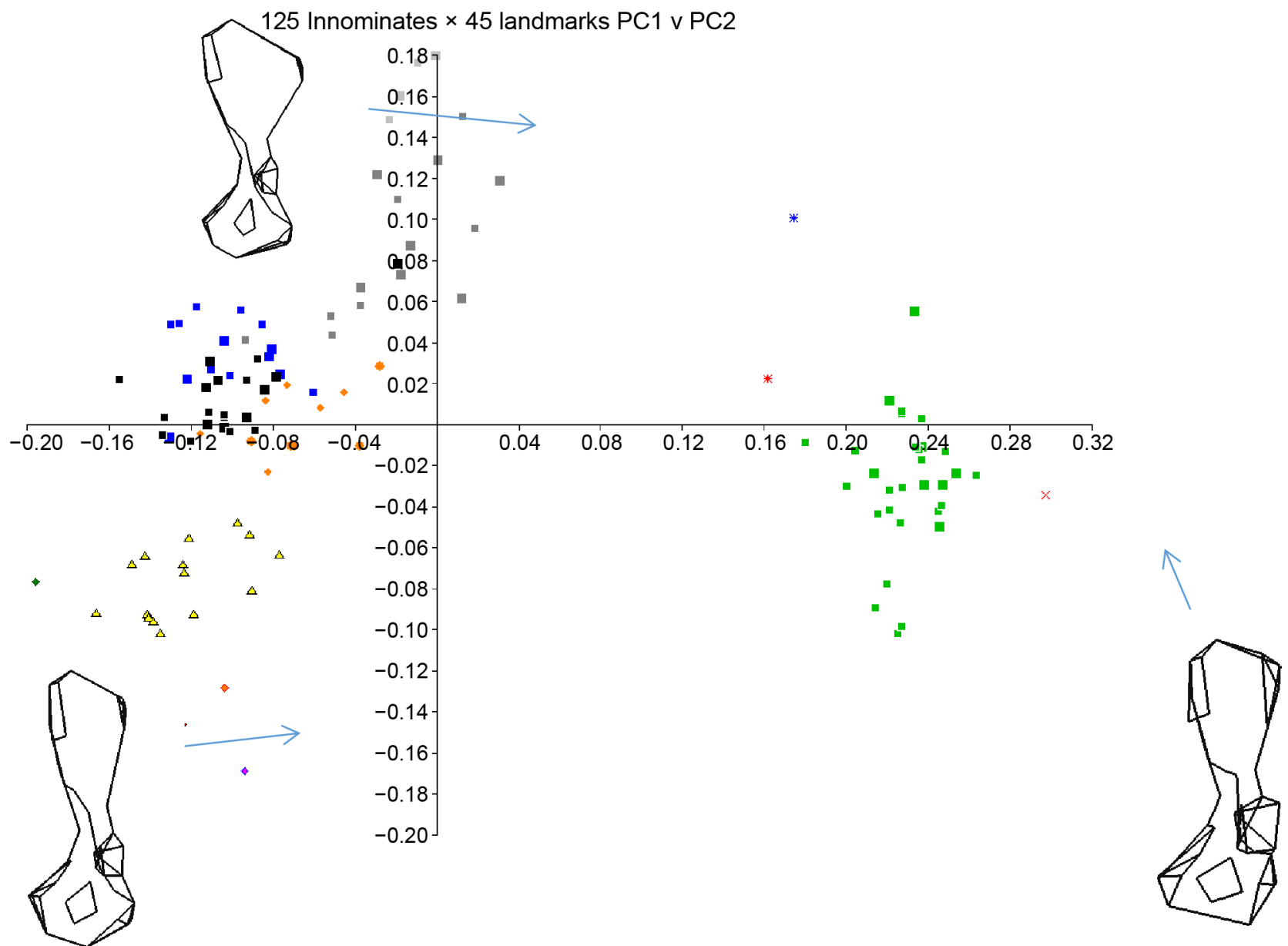

Figure 1. PC1 v PC2 of 125 Hip bones. 


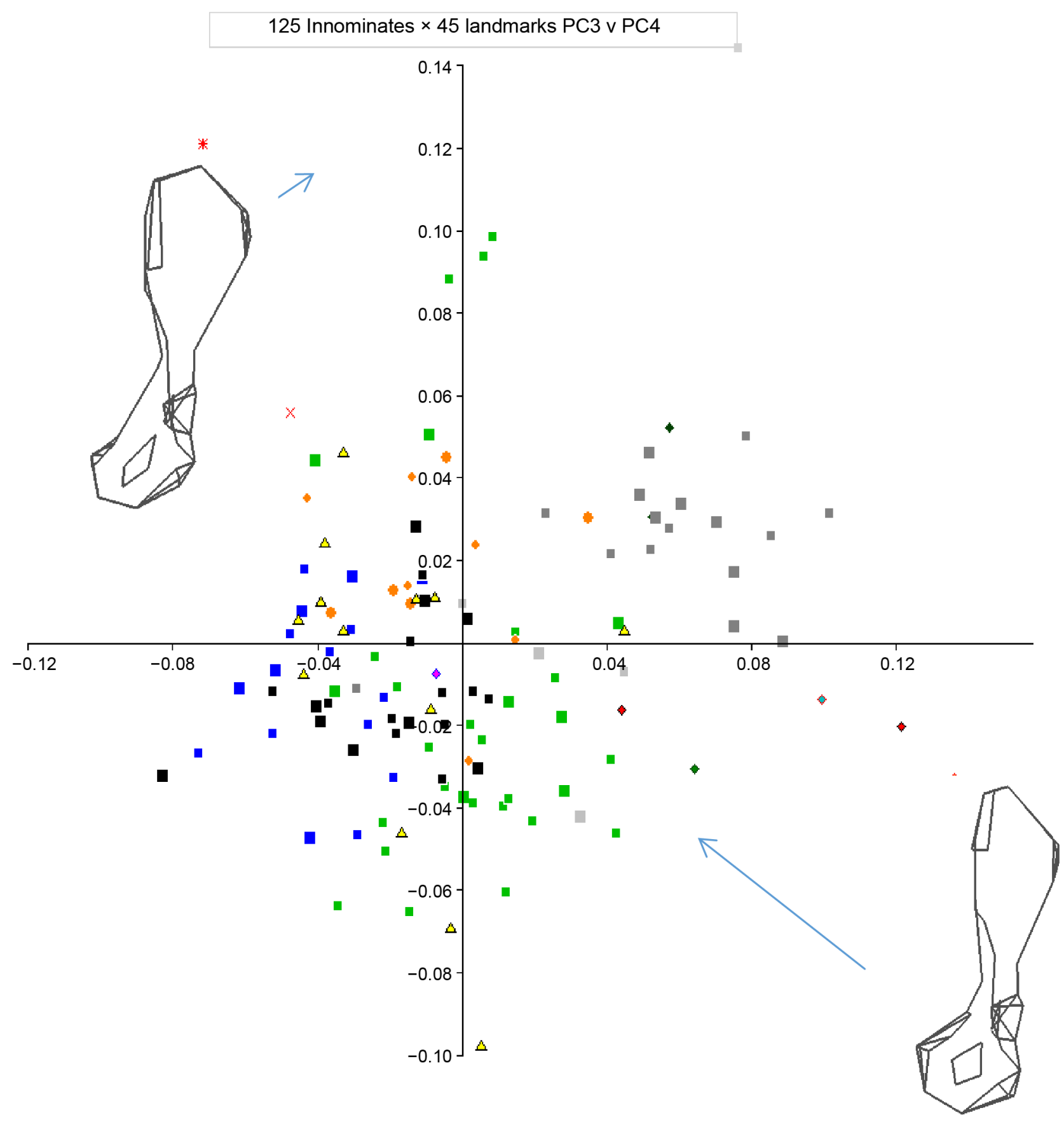

Figure 2. PC3 v PC4.

\subsubsection{Pelvis}

The shape analysis of 125 morphs of 19 species using 45 Hip bone landmarks is shown in Figure 1. Note that Morphologika not only plots the individual samples against two Principal Components, it also allows you to visualise an estimate of shape of the sample at any point on the chart. In the following figures, sample wire-frame diagrams of the sample are shown on the chart to give an impression of the average shape at a point on the chart indicated by a blue arrow. 
102 Sacra 19 sp

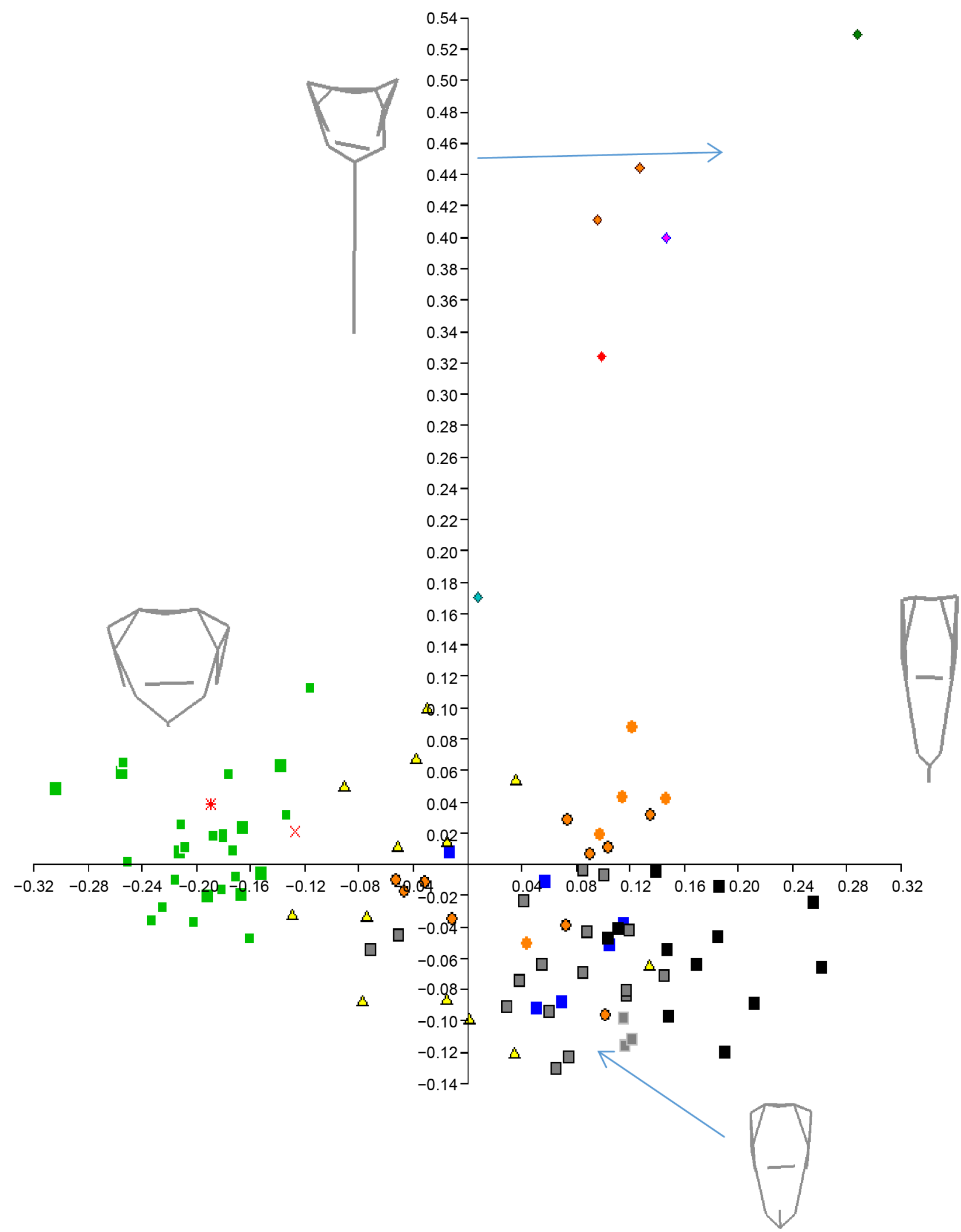

Figure 3. PC1 v PC2 Sacra. 


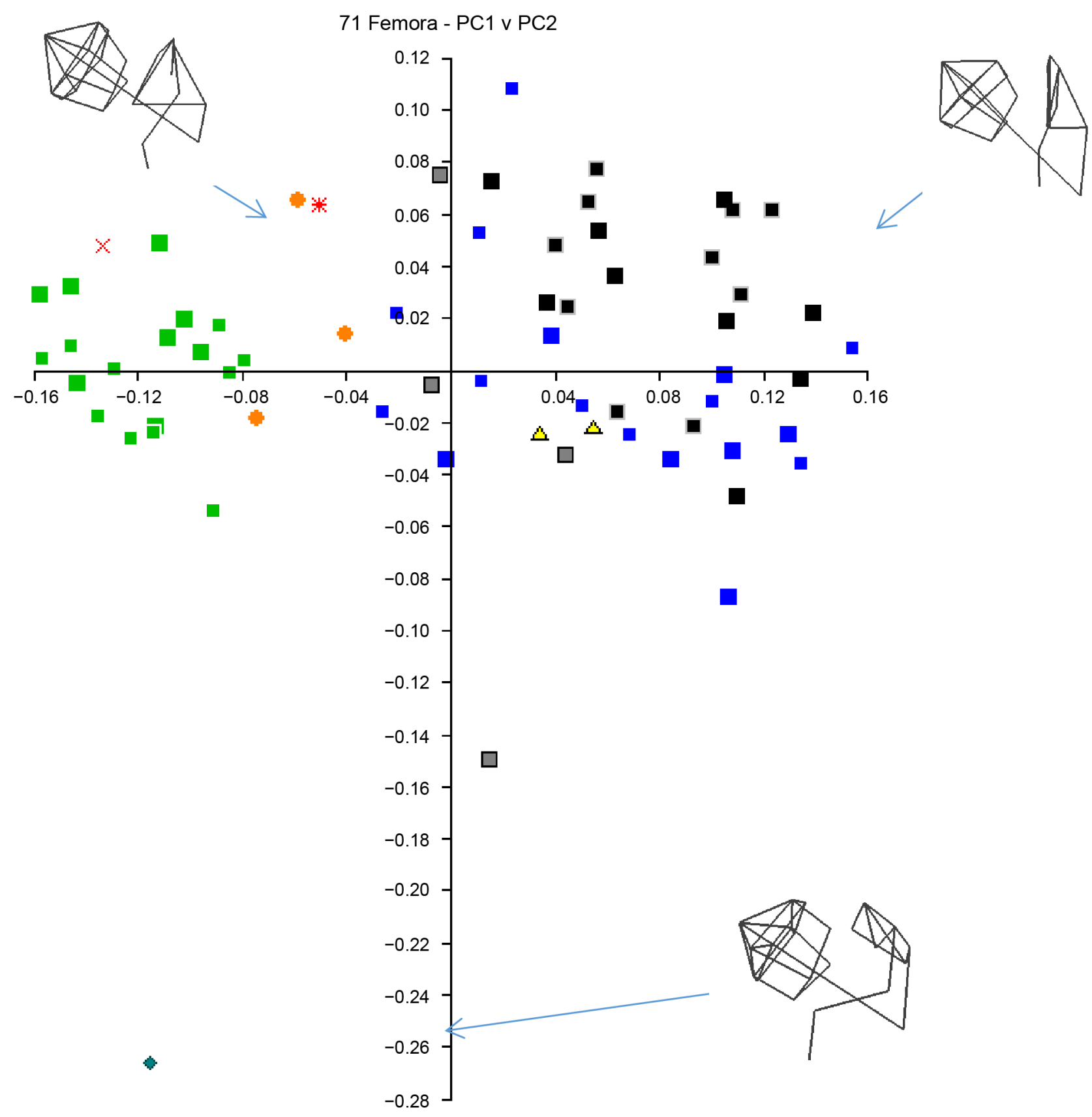

Figure 4. PC1 v PC2 proximal femur.

The percentage eigenvalues of the 4 most significant principal components were:

\begin{tabular}{ccc}
\hline PC & Variation & Cumulative \\
\hline PC1 & $51.3 \%$ & $51.3 \%$ \\
PC2 & $12.7 \%$ & $64 \%$ \\
PC3 & $4.3 \%$ & $68.3 \%$ \\
PC4 & $3.08 \%$ & $71.4 \%$ \\
\hline
\end{tabular}


Over $71 \%$ of the variation were represented by these 4 PCs, the majority of which was represented by PC1.

PC1 represents a distortion of the overall shape, generally, of the hip bone from one elongated in the Superior-Inferior (S-I) axis and narrowed laterally, to the opposite, shortened in the S-I axis and widened laterally. This PC appears to correlate more specifically with widening/shortening of the ischium rather than ilium.

PC2 represents a specific widening/shortening of the width of the ilium. For example, at one extreme one finds mostly Gorilla with relatively broad ilia, at the other old world monkeys, with relatively narrow ones.

The key finding of note here is that the shape of the australopithecine hip bone, although lying closer to Homo than to the great apes, is still significantly different from it, especially in $A$. africanus. This implies that although the australopithecines were very likely to have been bipedal, it is unlikely that their mode of locomotion was exactly the same as ours.

\subsubsection{PC3 and PC4}

Principal Components 3 and 4 together make up only $7 \%$ of the variation (compared to over $51 \%$ for PC1) but it is interesting to note that Australopithecus afarensis appears an outlier on the two combined. This seems to represent a lateral twisting of the iliac blade with respect to the rest of the hip bone and an inferior shearing of the pubic region including the pubic tubercle. Again this implies that australopithecines probably adopted a different gait to our own.

\subsubsection{Sacrum}

Not all the specimens measured included sacra, notably the paleospecies Australopithecus africanus.

\begin{tabular}{ccc}
\hline Percentage & Variation & Cumulative \\
\hline PC1 & $34.2 \%$ & $34.2 \%$ \\
PC2 & $21.8 \%$ & $56.0 \%$ \\
PC3 & $11.4 \%$ & $67.4 \%$ \\
PC4 & $6.3 \%$ & $73.7 \%$ \\
\hline
\end{tabular}

Australopithecus afarensis clustered well within the range of Homo sapiens on the main principal components indicating a similar shape and weight bearing function.

\subsubsection{Proximal Femur}

As the key paleo species in this study Australopithecus afarensis only contains a sample of its proximal femur, only the landmarks representing that part of the bone are analysed here. 


\begin{tabular}{ccc}
\hline Percentage & Variation & Cumulative \\
\hline PC1 & $24.4 \%$ & $24.4 \%$ \\
PC2 & $11.0 \%$ & $35.4 \%$ \\
PC3 & $8.9 \%$ & $44.3 \%$ \\
PC4 & $8.1 \%$ & $52.4 \%$ \\
\hline
\end{tabular}

$52.4 \%$ of the variation was found in the first 4 principal components.

The shape of the proximalfemur of Australopithecus afarensis clustered close to that of Homo sapiens in the two major principal components this analysis, along with the genus Pongo.

\subsubsection{Summary}

The shape analysis of sacrum and proximal femur generally clustered australopithecines close to, if not within, the normal range for Homo sapiens for those traits characteristic of bipedalism pertaining to weight bearing.

They differed somewhat in the shape analysis of the hip bone. The significant twisting of the ilium, with respect to the ischium, is quite different in australopithecines compared to other apes and their remarkable platypelloid shape is very different from Homo. These characteristics suggest that australopithecines have differences pertaining to muscle action and therefore perhaps are indicative of locomotor differences as first shown by the findings of Zuckerman et al. (1973) and Ashton et al. (1981).

This is not a remarkable observation, as it is pretty obvious from even a cursory glance at the australopithecine, Pan and Homo pelves (see Figure 5), especially in the context of the general Primate form which is, of the three, more chimp-like. In terms of the ratio between the lateral diameter and the A-P diameter, australopithecines and Pan are at the extremes, with Homo intermediate.

The first part of the 3D GM study of the shape of the australopithecine hip bone thus falsified the first null hypothesis listed earlier, concluding that the australopithecine pelvis differed markedly from that of Pan and Homo sufficient to question if adopted a mode of bipedal locomotion that was significantly differed from ours.

The next part of the study set out to investigate the muscle lever arms involved at the hip to try to identify those muscle blocks which were likely to have
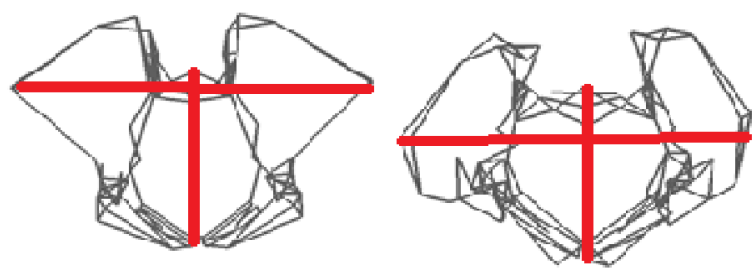

Lateral: Anterior-Posterior Ratio

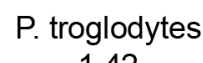

1.42

$$
\begin{gathered}
H . \text { sapiens } \\
1.65
\end{gathered}
$$

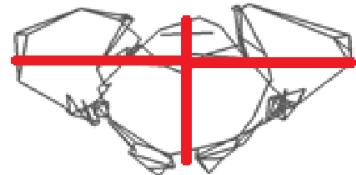

A. afarensis 2.65

Figure 5. Lateral: Anterior-Posterior diameter ratios (figures from MorphDb data set). 
been favoured most by the australopithecine skeletal anatomy. In this way, it might be possible to discern the kind of gait that australopithecines may have adopted, and how it might have differed from our own.

\section{Relative Muscle Lever Arm Study}

Of the three bones analysed for shape, only the hip bone showed major deviation in the australopithecine form from that of the genus Homo. The femur and sacrum did not show such marked deviation. The next logical step in this study, therefore, was to analyse the shape differences of the hip bone between the australopithecines and the genus Homo from the perspective of muscle action.

The objective was to analyse all the muscle lever arms involved in the movement of the hip, and to compare them against each other in order to try to identify those muscle blocks which were likely to have been favoured most by the anatomy of australopithecine hip and therefore the nature of the gait they adopted. Put simply, it will test the assumption, arriving from even a cursory glance at the remarkably platypelloid shape of the australopithecines pelvis, that their postcranial skeletal anatomy would appear better adapted to adduction/abduction of the thigh, relative to humans and chimpanzees.

See Supplementary Materials for a thorough description of the method used for all the muscle origins and insertion points.

\subsection{Results}

The "data mining" technique, commonly used in the world of business and commerce by using software such as Microsoft Excel and tools included in it like Pivot Tables, allows a vast amount of data to be summarised in a few lines of data. From these summaries, interesting data can be "drilled through" to get to the detail of what is going on behind the big picture.

This was the approach used here to analyse the large data set produced by this study-more than 135,000 lever arm ratios.

\subsection{Top Level Summary}

The overall averages of the permutations of lever arms grouped by muscle action for australopithecines as compared with Homo sapiens are summarised in the table below-in the cells above and to the right of the diagonal. Below and to the left are the relative rank order of Pan troglodytes relative to Australopithecus afarensis and Homo sapiens, among the 12 species analysed.

\begin{tabular}{|c|c|c|c|}
\hline & \multicolumn{3}{|c|}{ Australopithecus $\mathrm{v}$ Homo sapiens Ratio } \\
\hline & Flexion/Flexion & Rotation & Abduction/Adduction \\
\hline Flexion/Extension & & 0.730 & 0.754 \\
\hline Rotation & $12,11,1$ & & 0.997 \\
\hline \multirow[t]{3}{*}{ Abduction/Adduction } & $12,5,1$ & $5,2,4$ & \\
\hline & \multicolumn{3}{|c|}{ Australopithecine, Pan troglodytes, } \\
\hline & \multicolumn{3}{|c|}{ Homo sapiens ranking out of 12 species } \\
\hline
\end{tabular}


So, for example, the ratio of the mean lever arm values for muscles associated with Flexion/Extension for australopithecines is approximately $75 \%$ of that for Homo shown in the red cell at the top right.

When compared to the 12 species analysed, australopithecines ranked lowest for this ratio, Homo highest and Pan troglodytes ranked $5^{\text {th }}$-shown in the yellow cell in the bottom right.

The key finding is that the lever arms of muscles involved in flexion and extension, compared to those involved in rotation or abduction and adduction, are most different between Homo sapiens and australopithecines. The Australopithecus specimen AL 288-1 had an average lever arms ratio figure that was approximately $75 \%$ of that for Homo sapiens. It was ranked lowest of the twelve species studied, whilst Homo sapiens was ranked $1^{\text {st }}$.

When lever arms for muscles pertaining to adduction and abduction are contrasted with those involved with rotation, there is relatively little difference between Homo sapiens and australopithecines, both ranked in the middle of the list of species.

\section{3. $2^{\text {nd }}$ Tier Summary}

Drilling down on those two data points, one can see the figures in context of the full set of species and more statistical information about them.

\subsubsection{Extension/Flexion against Rotation}

The figure 0.730 in the table above is itself a ratio of two ratios for AL 288-1 and Homo sapiens. 0.908/1.243.

These figures each represent the average ratio of all permutations of lever arms of landmarks representing muscle origins involved with extension and flexion that are distinct from landmarks involved with rotation compared to those involved with rotation.

Table 2 shows similar figures from all the studied species and Figure 6 shows this data represented in order in a histogram.

The most striking point of note here is that Homo sapiens and AL 288-1 are at opposite ends of the ranked list of species.

The relatively high standard deviations inherent in these figures (e.g. Homo sapiens Mean $=1.243$; Standard Deviation $=0.553$ ) are due to the large variation of the landmark permutations used to make the extension/flexion and rotation muscle groups as the Pivot table generates an average for every landmark pair permutation.

\subsubsection{Extension/Flexion against Abduction/Adduction}

Similarly, the headline figure of 0.754 for extension/flexion compared to abduction/adduction in the summary table earlier can be "drilled through" in the same way, as shown in Table 3 and Figure 7.

Again, the distance between Homo sapiens and AL 288-1 is remarkable, considering both species are considered to be bipedal. The implication from these 
Table 2. Extension/Flexion v rotation.

\begin{tabular}{|c|c|c|c|c|c|c|}
\hline Rank & Row Labels & Min. & Avg. & Std. Dev. & Max. & $\mathrm{N}$ \\
\hline 1 & Homo sapiens sapiens & 0.432 & 1.243 & 0.553 & 3.747 & 588 \\
\hline 2 & Homo erectus & 0.567 & 1.156 & 0.605 & 3.012 & 28 \\
\hline 3 & Gorilla gorilla graueri & 0.477 & 1.112 & 0.493 & 3.167 & 168 \\
\hline 4 & Pongo pygmaeus & 0.495 & 1.106 & 0.506 & 2.474 & 56 \\
\hline 5 & Lemur catta & 0.429 & 1.106 & 0.548 & 2.327 & 28 \\
\hline 6 & Pongo abelii & 0.560 & 1.098 & 0.495 & 2.635 & 56 \\
\hline 7 & Hylobates moloch & 0.509 & 1.078 & 0.594 & 2.505 & 28 \\
\hline 8 & Pan paniscus & 0.285 & 1.066 & 0.484 & 2.712 & 504 \\
\hline 9 & Cercopithecus erythrotis & 0.594 & 1.037 & 0.447 & 2.073 & 28 \\
\hline 10 & Hylobates muelleri & 0.550 & 0.995 & 0.334 & 1.607 & 28 \\
\hline 11 & Pan troglodytes & 0.431 & 0.993 & 0.454 & 2.335 & 420 \\
\hline 12 & Australopithecus afarensis & 0.586 & 0.908 & 0.231 & 1.315 & 28 \\
\hline
\end{tabular}

Flexion/Extension : Rotation Lever Arm Ratio

Homo sapiens sapiens

Homo erectus

Gorilla gorilla graueri

Pongo pygmaeus

Lemur catta

Pongo abelii

Hylobates moloch

Pan paniscus

Cercopithecus erythrotis

Hylobates muelleri

Pan troglodytes

Australopithecus afarensis

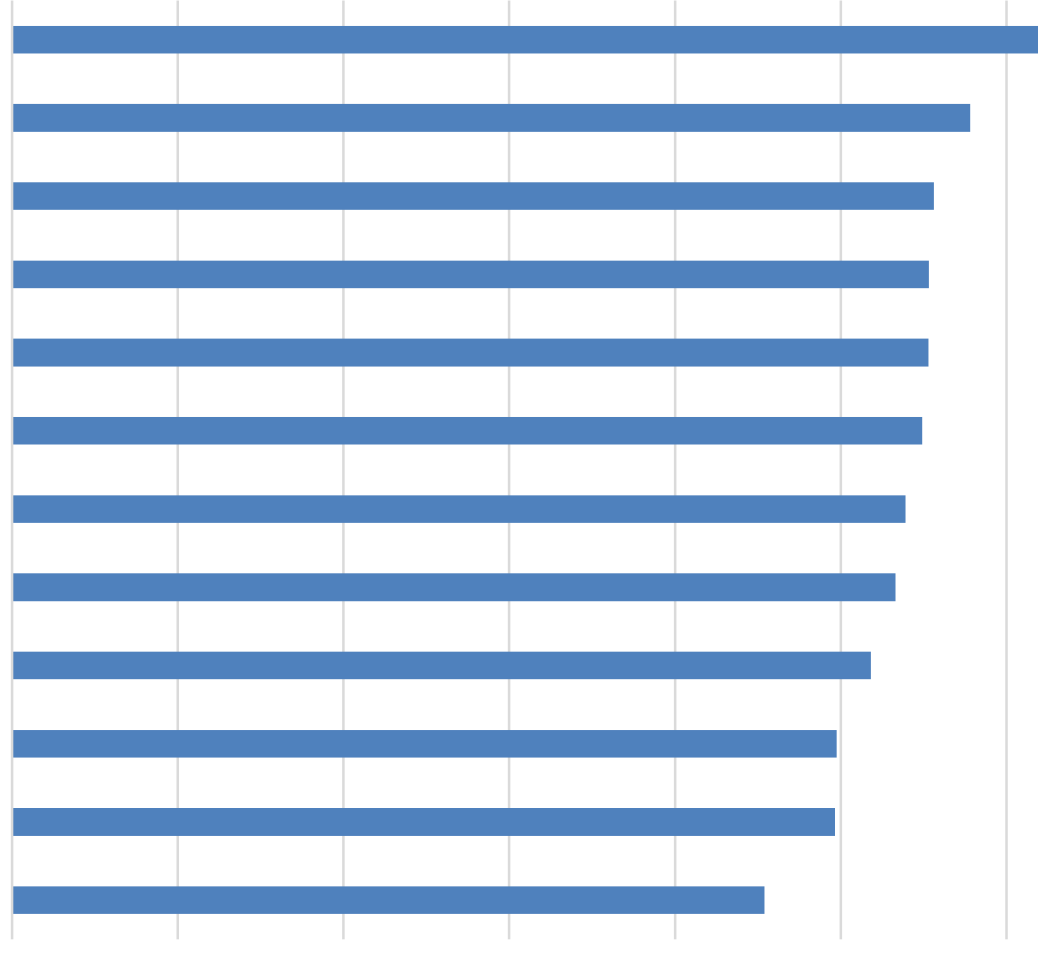

0.000

0.200

0.400

0.600

0.800

1.000

Figure 6. Extension/Flexion v rotation summary. 
Table 3. Extension/Flexion versus Adduction/Abduction.

\begin{tabular}{|c|c|c|c|c|c|c|}
\hline Rank & Row Labels & Min. & Avg. & Std. Dev. & Max. & $\mathrm{N}$ \\
\hline 1 & Homo sapiens sapiens & 0.626 & 1.408 & 0.567 & 4.242 & 672 \\
\hline 2 & Homo erectus & 0.625 & 1.399 & 0.640 & 3.488 & 32 \\
\hline 3 & Pongo pygmaeus & 0.449 & 1.246 & 0.582 & 3.178 & 64 \\
\hline 4 & Gorilla gorilla graueri & 0.516 & 1.239 & 0.577 & 4.298 & 192 \\
\hline 5 & Pan paniscus & 0.330 & 1.212 & 0.591 & 4.356 & 576 \\
\hline 6 & Pongo abelii & 0.518 & 1.212 & 0.563 & 3.483 & 64 \\
\hline 7 & Lemur catta & 0.620 & 1.198 & 0.550 & 2.528 & 32 \\
\hline 8 & Pan troglodytes & 0.394 & 1.155 & 0.594 & 3.392 & 480 \\
\hline 9 & Hylobates moloch & 0.324 & 1.108 & 0.731 & 3.640 & 32 \\
\hline 10 & Cercopithecus erythrotis & 0.479 & 1.103 & 0.551 & 2.717 & 32 \\
\hline 11 & Hylobates muelleri & 0.521 & 1.093 & 0.423 & 2.077 & 32 \\
\hline 12 & Australopithecus afarensis & 0.659 & 1.061 & 0.296 & 1.871 & 32 \\
\hline
\end{tabular}

\section{Flexion/Extension : Abduction/ Adduction Lever Arm Ratio}

Homo sapiens sapiens

Homo erectus

Pongo pygmaeus

Gorilla gorilla graueri

Pan paniscus

Pongo abeli

Lemur catta

Pan troglodytes

Hylobates moloch

Cercopithecus erythrotis

Hylobates muelleri

Australopithecus afarensis

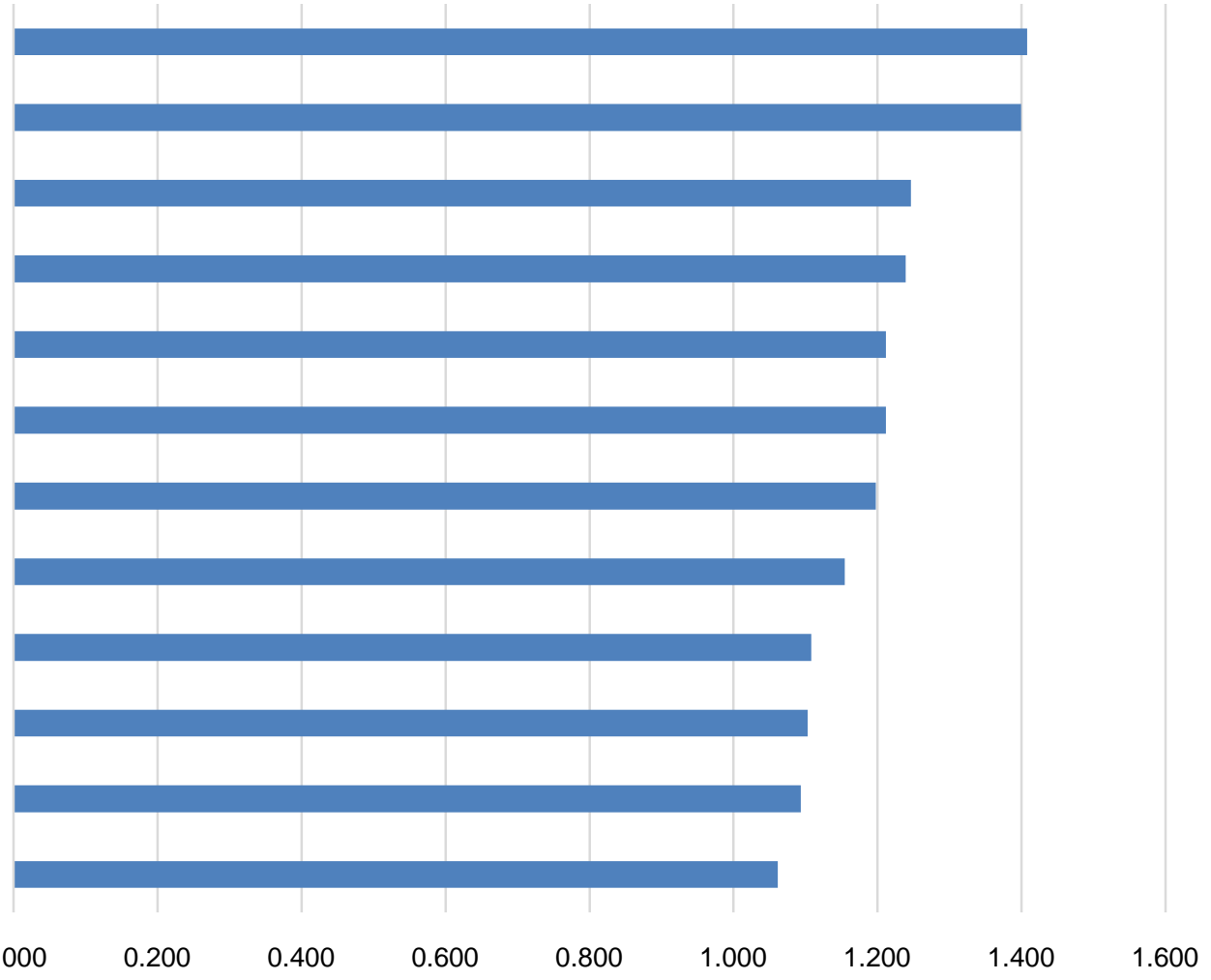

Figure 7. Extension/Flexion versus Abduction/Adduction Summary. 
data is that the shape of the australopithecine pelvis must have been adapted to a different locomotor repertoire than the modern human form.

\section{4. $3^{\text {rd }}$ Tier Summary}

Drilling down further, one might next tease out more specific muscle groupings, some which give even clearer results, and others which give more ambiguous findings. For example flexion and extension can be split apart and compared with either lateral or medial rotation, or with adduction and abduction, individually.

Taking one example, if one compares lever arm ratios for muscles exclusively involved with flexion with those exclusively involved in medial rotation, one finds an even greater difference between Homo sapiens and AL 288-1.

Here the ratio is $0.648(1.082 / 1.669)$. Below, in Table 4 is similar data for all the species and, again, it is summarised in Figure 8.

By contrast, if one compares the lever arms for extension with those for adduction, one finds very little difference between Homo sapiens and AL 288-1 (Ratio 0.951).

Detail, again, shown below in Table 5 summarised in Figure 9.

As one can see, there is a vast number of permutations of pairs of landmarks and landmark groups that could be used to perform analysis with this data. But considering the significant caveats about the data outlined earlier, perhaps it would be better to end at this point, to step back from the detail a little and to discuss what the results may or may not indicate.

Table 4. Flexion versus medial rotation.

\begin{tabular}{|c|c|c|c|c|c|c|}
\hline Rank & Row Labels & Min. & Avg. & Std. Dev. & Max. & $\mathrm{N}$ \\
\hline 1 & Homo sapiens sapiens & 0.635 & 1.669 & 0.641 & 3.747 & 168 \\
\hline 2 & Lemur catta & 0.753 & 1.589 & 0.531 & 2.327 & 8 \\
\hline 3 & Homo erectus & 0.664 & 1.581 & 0.769 & 3.012 & 8 \\
\hline 4 & Pongo pygmaeus & 0.535 & 1.481 & 0.587 & 2.474 & 16 \\
\hline 5 & Gorilla gorilla graueri & 0.685 & 1.480 & 0.541 & 3.167 & 48 \\
\hline 6 & Pongo abelii & 0.666 & 1.449 & 0.577 & 2.635 & 16 \\
\hline 7 & Pan paniscus & 0.413 & 1.427 & 0.509 & 2.712 & 144 \\
\hline 8 & Hylobates moloch & 0.608 & 1.411 & 0.680 & 2.482 & 8 \\
\hline 9 & Cercopithecus erythrotis & 0.604 & 1.349 & 0.487 & 2.073 & 8 \\
\hline 10 & Pan troglodytes & 0.498 & 1.312 & 0.468 & 2.335 & 120 \\
\hline 11 & Hylobates muelleri & 0.679 & 1.279 & 0.357 & 1.607 & 8 \\
\hline 12 & Australopithecus afarensis & 0.702 & 1.082 & 0.246 & 1.315 & 8 \\
\hline
\end{tabular}




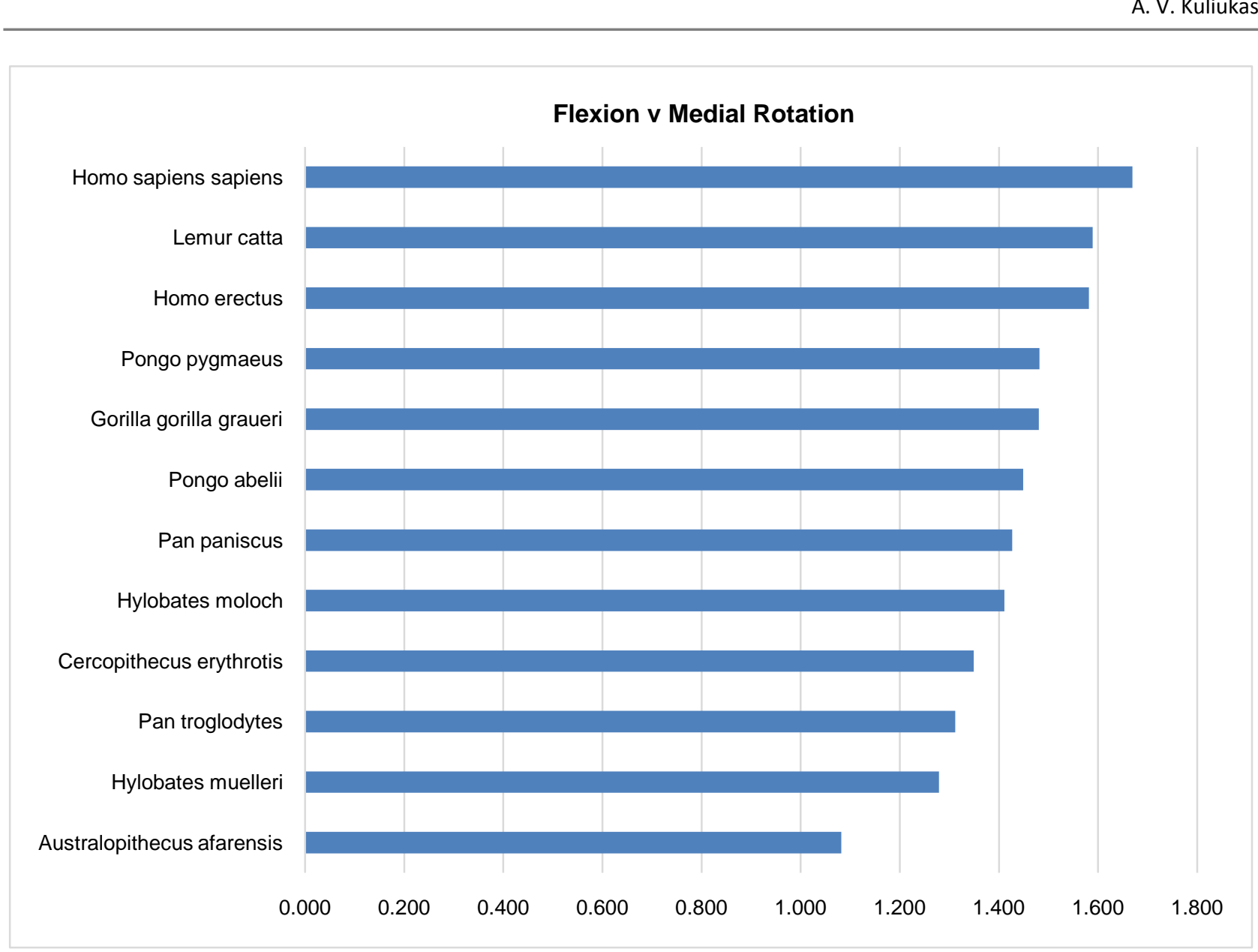

Figure 8. Flexion versus medial rotation summary.

Table 5. Extension versus adduction.

\begin{tabular}{|c|c|c|c|c|c|c|}
\hline Rank & Row Labels & Min. & Avg. & Std. Dev. & Max. & $\mathrm{N}$ \\
\hline 1 & Homo sapiens sapiens & 0.512 & 1.264 & 0.461 & 3.617 & 1155 \\
\hline 2 & Australopithecus afarensis & 0.687 & 1.202 & 0.442 & 2.146 & 55 \\
\hline 3 & Homo erectus & 0.513 & 0.940 & 0.226 & 1.632 & 55 \\
\hline 4 & Gorilla gorilla graueri & 0.483 & 0.903 & 0.259 & 1.871 & 330 \\
\hline 5 & Pongo abelii & 0.487 & 0.877 & 0.235 & 1.656 & 110 \\
\hline 6 & Pongo pygmaeus & 0.462 & 0.867 & 0.268 & 1.916 & 110 \\
\hline 7 & Hylobates muelleri & 0.530 & 0.760 & 0.175 & 1.279 & 55 \\
\hline 8 & Pan paniscus & 0.330 & 0.759 & 0.213 & 2.176 & 990 \\
\hline 9 & Lemur catta & 0.510 & 0.720 & 0.150 & 1.141 & 55 \\
\hline 10 & Pan troglodytes & 0.392 & 0.681 & 0.162 & 1.520 & 825 \\
\hline 11 & Cercopithecus erythrotis & 0.473 & 0.628 & 0.134 & 1.021 & 55 \\
\hline 12 & Hylobates moloch & 0.327 & 0.530 & 0.188 & 0.915 & 55 \\
\hline
\end{tabular}




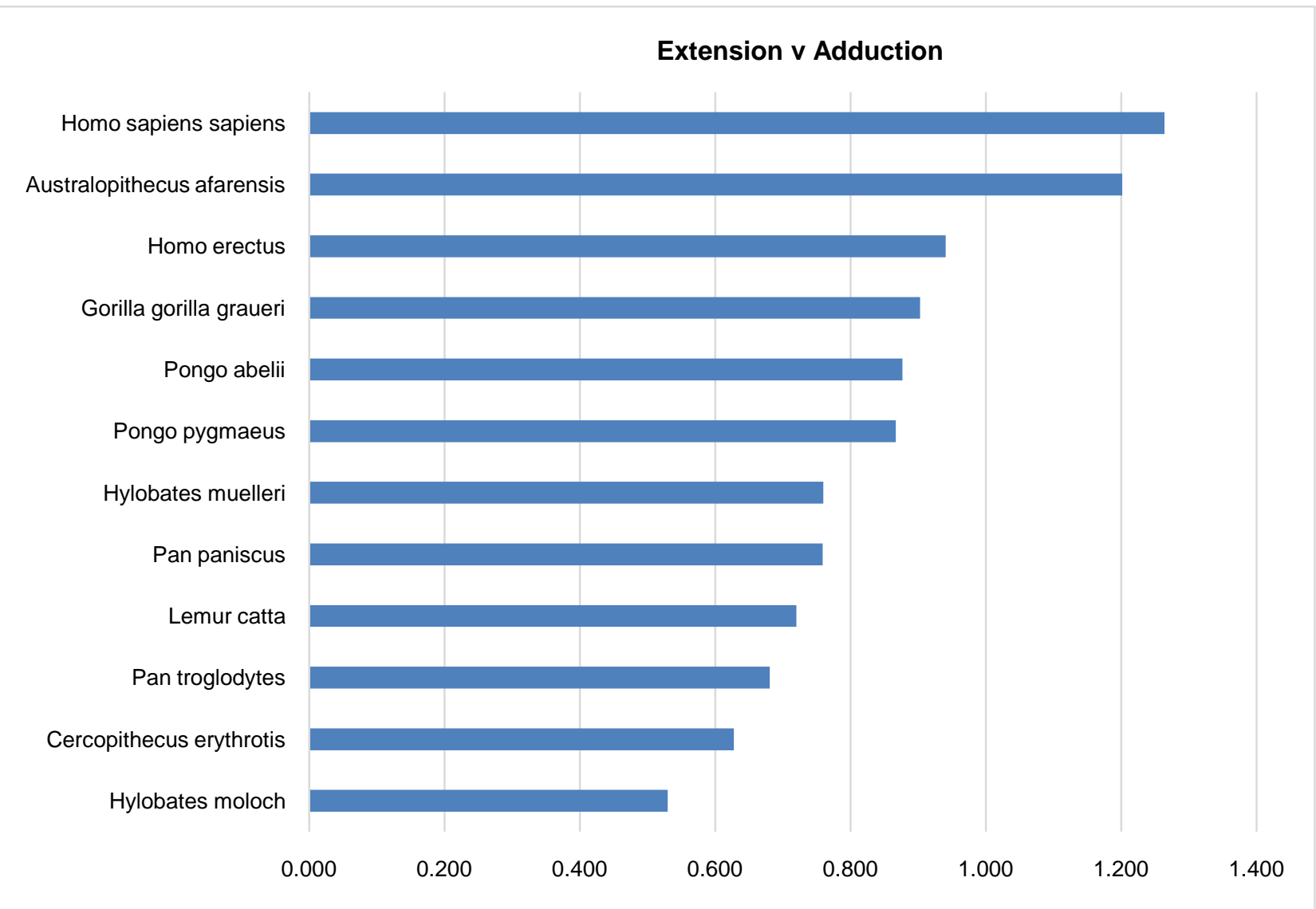

Figure 9. Extension versus adduction summary.

\section{Discussion}

\subsection{Australopithecines Had Differently Shaped Pelves to Humans}

A 3D GM analysis of the shape of the hip bone, sacrum and femur was consistent with earlier studies that indicated that australopithecines were similar to humans in many aspects pertaining to bipedalism, and supported the long-held conviction that they were almost certainly obligate bipeds, like us. The shape of the australopithecine proximal femur and sacrum, in particular, clustered well within that of Homo sapiens. However, consistent with other studies (e.g. Zuckerman et al., 1973), it was shown that sufficient differences in the shape of the hip bone between the AL 288-1 specimen and Homo sapiens were identified to indicate that australopithecines might have used a bipedal gait that was quite unlike the striding, "inverted-pendulum" gait of modern humans.

\subsection{Lever Arm Analysis Indicates a Greater Utility for Adduction/Abduction in Australopithecines}

A more detailed investigation of load arms of potential muscle origins, grouped by major muscle action, was consistent with this and identified differences in lever arms in muscles pertaining to flexion in particular, as compared to rotation and abduction/adduction of the hip, as being largest between humans and aus- 
tralopithecines in the species studied.

Clearly, many individual muscle lever arm ratios could be analysed to increase the depth of this study and to derive quantitative data but even this limited study shows approximately a $25 \%$ difference in the lever arm ratio between muscles involved with flexion/extension compared to those involved with abduction/adduction and rotation.

\subsection{Could These Features Be Explained as Energy-Efficiency Adaptations?}

The classic interpretation of the australopithecine pelvis is that it was not only a biped but an efficient one. Indeed, according to Don Johanson's anecdote in his book "Lucy-The Beginnings of Humankind", all he had to do was to show the fragments of the knee joint he had collected from Hadar to Owen Lovejoy and he pronounced that had come to that very conclusion, immediately. To Johanson's question about its bipedality, Lovejoy apparently replied "My friend, he could walk upright. Explain to him what a hamburger was, and he'd beat you to the nearest McDonald's nine times out of ten" (Johanson \& Edey, 1981: p. 163).

The anatomical feature Lovejoy was identifying was the bicondylar angle, which is indeed interpreted as being a trait indicative of an energy efficient gait. Australopithecines have a bicondylar angle that is greater than humans and this is interpreted as being in accordance with the fact that they had relatively wide pelves and relatively short lower limbs.

In humans the bicondylar angle indicates the placing of the knee directly under the center of mass during the stance phase (to reduce energetically costly swaying from side to side) and, if australopithecines had wider hips and shorter limbs, then this angle would need to be all the more to achieve the same thing.

There is also the evidence of the distal femur (from a different specimen) that is indicative of fully extended bipedalism - the condyles are more ovoid in shape from the lateral aspect than those of extant great apes, a trait interpreted as being an adaptation for walking with an extended knee, consistent with a striding gait, like modern humans.

However, there are some key problems with this interpretation that seem to have been skipped over.

The very short stature and short lower limbs of australopithecines are contra-indications to an efficient striding gait, even if they were walking on optimal surfaces: those that are flat, firm and vegetation-free. However the Hadar paleohabitat evidence appears to show almost the opposite. Far from being dominated by flat, open grassland, it appears to have been rather wet and wooded. Indeed it seems more likely to have been a wetland for a million or so years around the time of death of AL 288-1 (Johanson \& Edey, 1981: p. 129).

\subsection{Putative Side-To-Side Wading Gait}

It is interesting to speculate about what form of locomotion might best account 
for such a radically different hip bone shape and to consider which type of muscle action would best be biomechanically favoured by the australopithecine hip as compared to the human form.

If the australopithecine pelvis indicates bipedality, but not our kind of bipedality, it begs the question: What other kinds might there be?

As the morphology of the australopithecine hip is most certainly unlike most of the (largely arboreal) more primitive forms analysed here, it seems unlikely that such a departure from the Primate "norm" could be explained by a peculiar combination of exclusively terrestrial and arboreal locomotion as suggested by Oxnard \& Hoyland-Wilkes (1994: p. 22).

It is argued here that the simplest and most satisfactory potential explanation for their pelvic shape anomalies is a significant wading component in their locomotor. A twisting, side-to-side gait (one perhaps likely in waist deep water) would be easier to perform with a hip which gave biomechanical advantage to rotation, abduction and adduction of the hip. The remarkably platypelloid shape of the australopithecine pelvis (Tague \& Lovejoy, 1986) might similarly be explained as a streamlining adaptation to moving though water using such a twisting, side-to-side gait as illustrated in Figure 10.

\subsection{Drag Reduction and Pelvic Shape}

Motion in water incurs far greater drag forces than in air and so it is logical that any hominid adapted to significant amounts of wading would be expected to have evolved traits to reduce that drag. In fluid dynamics, the drag coefficient is

Postulated Side-to-Side

Wading Gait

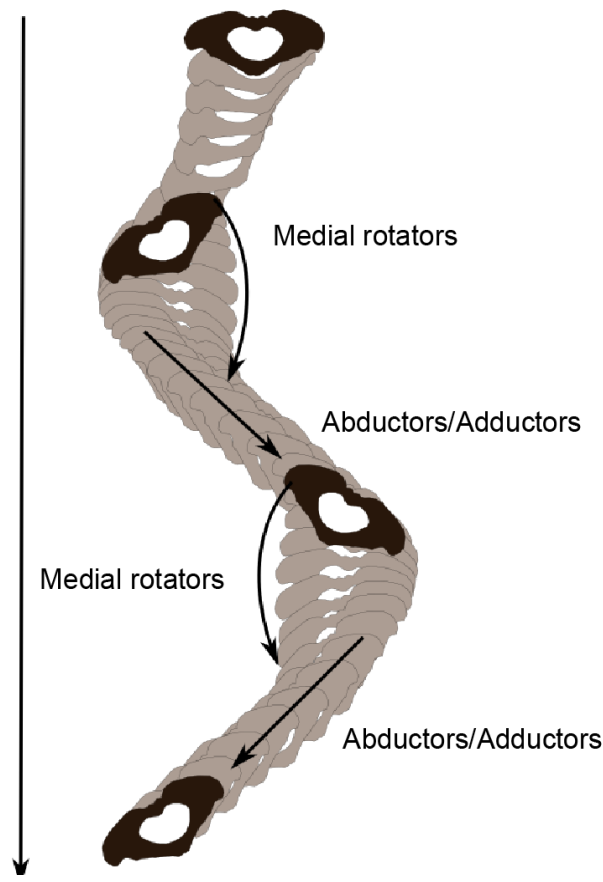

Figure 10. Postulated side-to-side "wading" gait. 
usefully estimated to determine the force of drag that an object will be subjected to as it moves. The drag coefficient is associated with its surface area in the plane of motion and is contributed by two effects: skin friction and form drag. The degree of form drag is heavily dependent on the shape of the object. Classic "streamlined" shapes impart much lower drag coefficients than ones that are not streamlined (see Figure 11 below).

The area of the lateral profile of the australopithecine at the hip must have been significantly less than one would expect if they had a pelvis shaped like most apes or large primates. As drag is calculated to be proportional to the area of the shape, it can be inferred that having a lateral profile that has half the area of, say, a chimpanzee could be expected to reduce drag by a significant amount (Kuliukas, 2001).

\subsection{Side-to-Side Gait Speculation "Explains" Lever Arm Differences}

The side-to-side gait postulated would also predict a greater biomechanical premium on those muscles involved in rotation and adduction/abduction of the thigh. It is argued here that no other explanation of the peculiarly platypelloid shape of the australopithecine pelvis has been so succinctly argued.

Of course, such speculation could be criticised as being fanciful and/or circular: One layer of speculation placed on top of another. However, the fact remains that the australopithecine pelvis does indicate a form of bipedalism that is not quite like ours and it as form that is remarkably different from the "prototype" Primate form, one clearly strongly associated with arboreality.

\subsection{Extant Ape Wading Behaviour Support}

If one ties this in with anecdotal evidence of observations of extant great apes moving in shallow water (see e.g. Karlowski, 1996; Bearder, 2000; Tutin et al., 2001; Myers-Thompson, 2002; Kuliukas, 2002; Breuer et al., 2005), the idea is greatly supported. There is no other scenario in the natural world where otherwise committed quadrupedal great apes will so predictably switch to moving (not just posing momentarily) bipedally-and remain doing so as long as the conditions prevail - than in waist deep water.

\subsection{Evaluating Predictions of the Wading Hypothesis}

To the two falsifiable null hypotheses listed earlier to test the wading hypothesis, a third might be added here:

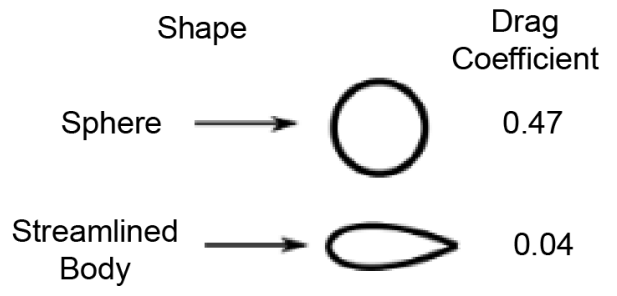

Figure 11. Effect of shape on drag coefficient. 
- Any biomechanical advantage inferred by the anatomy of the australopithecine pelvis would not have given them any advantage whilst wading in waist deep water.

It is argued that the first two null hypotheses listed earlier have been falsified and that the third, listed above, if not falsified, appears inconsistent with what these results appear to show.

\section{Wading into Anthropology}

This paper has provided new empirical evidence that is consistent with the wading hypothesis of hominin bipedal origins. It is argued that the hypothesis works just as well whether the ancestors of all the great apes were already somewhat bipedal, or not. That is, if the genus Homo uniquely gained bipedalism, or the genera Pan and Gorilla both lost it. Wading in shallow water-or the withdrawal from it-seems to be a plausible scenario to help explain the origins of hominin bipedalism and/or why some hominins stopped using that form of locomotion.

Finally, a summary of the strengths of the wading hypothesis, compared to other models of hominid bipedalism, is provided, as this is a model that has rarely been discussed in the anthropological literature.

\subsection{An Evaluative Framework for Models of Bipedalism}

There are at least 30 distinct ideas about the evolutionary scenarios likely to have led to human bipedalism and they are described in a fairly voluminous literature (for a full review see Kuliukas, 2017 or http://www.tinyurl.com/BipedalModels). When a model or set of ideas is first described in the scientific literature, the paper begins with a summary of other ideas that have been published before. However, there never appears to be any systematic method for doing this. Usually, a small subset of the published ideas are quickly summarised by the author(s) before they begin in describing the evidence pertaining to their preferred model. Missing in the literature until recently, it would seem, is any attempt to systematically assess the models that have been published and come up with an evaluative framework against which all models might be rated.

Kuliukas, 2017 made such an attempt to provide such an evaluative framework, and compared all of the known published models on the origin of human bipedalism. In doing so, he noted the disparity between the apparent strength of wading hypothesis when contrasted with the lack of favourable reporting of it in university level text books.

This paper will therefore end with a summary of that evaluative framework as it relates to the wading hypothesis.

\subsection{Offers a Powerful Selective Force for Selection}

Of all the models of bipedal origins, only wading can argue, even in theory, that if a would-be hominin tried to move quadrupedally in the proposed scenario, it would die. 


\subsection{It Is Not Teleological}

Many of the models of bipedal origins use a rather obvious benefit of bipedality in modern humans as the proposed driver. "Carrying" models are a good case in point. There is no doubt that carrying is one clear benefit of bipedalism for us today, but it more difficult to conceive of scenarios where it could have driven the origin of bipedality. Wading does not suffer from such problems. Even extant apes, that are committed to quadrupedalism on dry land will predictably switch to bipedalism in waist deep water.

\subsection{Offers Improved Food Acquisition}

Several models argue that bipedality would have improved food acquisition in the proposed early bipedal hominin. Similarly, wading can make such claims as wet, swampy habitats are known to have a rich biomass, especially in times of drought. Wrangham et al. (2009) argued that bipedal wading in places like the Okavango delta could have been a significant component of bipedal origins.

\subsection{Accounts for Predation}

A good model of bipedal origins should either place hominins in relatively safe zone with few predators or at least offer a plausible counter argument to the issue of predation. Many savannah-based models can be criticised for this as they place vulnerable hominins, on the open plains, or adjacent to them-often whilst carrying meat or even more vulnerable infants. It could be argued that wading does this too, as there are several very dangerous aquatic predators in such habitats, notably crocodiles and hippopotami. However, the wading model proposes that the earliest hominins also were relatively arboreal and could have climbed trees to escape such predators. Also, some wading hypotheses propose coastal locations, where there are fewer predators.

\subsection{Explains Why Other Great Apes Are Not Bipedal}

A good model of hominin bipedal origins should not only account for our bipedality but also why chimpanzees and gorillas move quadrupedally as knuckle-walkers. This became particularly pertinent as fossil evidence emerged around 2000 that bipedalism was much older than we had thought, to the point that it may even precede the split with Pan and Gorilla. So the model should also be flexible enough to explain how the LCA might have been somewhat bipedal from which evolved one lineage that became more so, and two that "reverted" to quadrupedalism. Wading in swampy habitats offers the perfect scenario for this as even extant apes move bipedally in shallow water and being an "ex-wading" ape seems intuitively fitting as a way of explaining knuckle walking.

\subsection{Has Compelling Analogous Behaviour in Extant Apes}

Kevin Hunt $(1994,1996)$ suggested that scenarios where bipedalism is exhibited in extant apes might provide big clues as to the sort of situation where our an- 
cestors might have begun moving bipedally. Hunt's extensive study of wild chimpanzees found that $80 \%$ of instances of bipedalism were in the context of postural feeding which led him to propose that this would have been a plausible scenario for the origin of our bipedality.

However practically every such recorded incident was arboreal and all of them were with the support of their upper arms. Also, this postural form of bipedalism, in any case, did not comprise any form of locomotion.

It begs the question: Is there a different, perhaps rarer scenario where extant apes are compelled not just to pose bipedally momentarily, but to move on two legs without the support of the upper limbs. Clearly wading through shallow water provides precisely this scenario. Indeed it is difficult to envisage any other scenario where a group of extant apes could be induced to move bipedally forever as long as the conditions prevailed.

\subsection{Applies to Both Sexes}

Some models of the evolution of bipedality argue that it was somewhat driven by one gender. Hunting models are one example of this, including the recent fashion to promote endurance running as a significant factor of the evolution of human bipedality. They are not all male-biased. Some promote young mothers carrying infants as a key driver, or even female-driven sexual selection based on penile display.

There could, of course, be an element of truth in such ideas but I would argue that, all things being equal, a model of hominin bipedality that worked equally for males and females should be considered stronger than one that didn't. In this regard then, of course, wading meets the criterion.

\subsection{Explains Anomalies of the Australopithecine Postcranial Skeleton}

One of the motivations for writing this paper was the fact that very few papers have been written about the peculiar shape of the australopithecine pelvis. It's extremely platypelloid is unique amongst the Primates, most species of which have almost the exact opposite ratio of A-P: lateral dimensions. Although this has been widely interpreted as being symptomatic of bipedality, few seem to have focused on what the platypelloid shape might tell us about how they moved and attempted to derive a model of bipedal origins around such scenarios.

One notable exception in John Kingdon's (2003) "Squat feeding" hypothesis which goes into some detail about the shape of their pelvis and argues that living on the (outside) edge of gallery forests in woodland that started to open up into surrounding savannah grasslands there would be the possibility of collecting food that fell from trees, such as nuts and fruits, and this would have led to the phenomenon of "squat feeding" - a neat precursor to bipedality.

This paper provides compelling complementary evidence to part of Kingdon's idea. Gallery forest habitats do indeed seem to have been inhabited by early bi- 
pedal hominins. However, rather than the pertinent scenario being on the outside margins of the gallery forest, this paper argues that the inner margins-i.e. close to or in the shallow water near the banks-offer a far stronger and more plausible scenario. A wading gait that involved a degree of lateral motion, perhaps with a side-to-side twisting motion, appears to offer a perfectly plausible adaptive explanation for the australopithecine pelvic shape. Its platypelloid shape would offer significant drag reduction whilst moving through water laterally and the shape of the pelvis generally seems to favour the muscle groups that would propel such wading - increased adduction, abduction and rotation.

\subsection{Consistent with the Palaecological Record}

One of the most obvious aspects of a plausible model of human bipedality is that it should be supported by strong evidence from the fossil record. At least the fossil evidence should consistent with it. So if, for example, the model promotes some aspect of life on the open plains of Africa was the driver of human bipedality, one would expect to find fossil evidence of putative early bipeds in geographic locations, in paleohabitats, consistent with that model.

Bearing in mind the taphonomy of the process of fossilisation suggests that most fossils are formed in depositional substrates, it is almost tautological that waterside habitats conducive to wading are likely to have been present when hominids died and became fossilised.

On top of this self-evident link, however, there are several key examples of the paleohabitats of early bipeds that appear especially conducive to the sort of swampy wetlands one would predict to be associated with wading-climbing apes. Sahelanthropus. Hadar. To name but two.

\subsection{Offers a Plausible Precursor to Both Knuckle-Walking and Striding Bipedalism}

Very few of the published models on the origin of hominid bipedalism concern themselves with the sister problem-explaining the equally peculiar mode of locomotion of our great ape cousins, the chimpanzees and gorillas. Striding bipedalism is unique in the animal world, as is knuckle walking. It seems to me that a really good model of bipedal origins should explain both, simultaneously. If one considers bipedal origins as a very early phenomenon preceding the split of the Homininae, Paninae and Gorillinae and that this last common ancestor already practiced a kind of wading-climbing facultative bipedalism. I have outlined here how I consider wading-climbing to be an ideal precursor for striding bipedalism, but it is also my contention that is also works rather well as a precursor to knuckle-walking too, if one considers a kind of "ex-biped" whose locomotor repertoire now involves less wading than before but more locomotion through dense wooded vegetation.

\subsection{It Is Complimentary to Other Models}

There is so much overlap between the different published ideas on the origin of 
hominin bipedalism that it is difficult to say, with any accuracy, even how many models there are. However, an objective audit would reveal at least 20 distinct ideas and possible more than 40 . Most of these ideas on bipedal origins have been published by reputable scientists who have considered the problem for years. It seems unlikely, then, that any single idea will prove to be absolutely correct rendering all the others absolutely wrong. Much more likely that whatever actually drove hominid bipedality included elements of all most, if not all, of the models. One criterion, then, that one might expect to be a key component of a good model of bipedal origins is how complimentary, or at least compatible, it is with the others.

An analysis of the models shows that climbing models and carrying ones appear to be a little contradictory as one cannot carry and climb at the same time. But it could be argued that wading models are complimentary to most.

\subsection{Offers Testable Predictions}

Few of the published models of bipedal origins seem to be phrased in a strict, Popperian, scientific phraseology. Specifically, few make testable predictions and set out to test them. It was with this criticism in mind that I set out to make predictions of the wading hypothesis, as well as come up with a set of objective criteria to criticise them. I argue that the wading hypothesis, as defined and tested here, meets this criterion as well as any other.

\subsection{Has Extended Explanatory Power}

A final attribute of a good model of bipedal origins is that it should also be consistent with, or complimentary to, ideas that explain the whole gamut of human phenotypic traits that differ from the great apes. It is not only in our mode of locomotion that we differ. We also have undergone a clear process of encephalisation and dental/masticatory reduction that seem to indicate a distinctive shift in diet. The body hair pattern of humans also differs significantly from chimpanzees and gorillas. Although we are not exactly "naked apes"-our follicle density is similar to that of the great apes-there is no question that the pattern of body hair-large areas covered with very fine vellus hairs as opposed to terminal hairs-differs greatly. Perhaps most remarkable of all is the human predisposition to complex grammatical language. Although there remains a great deal of controversy and criticism about the so-called "aquatic ape hypothesis" (Hardy, 1960; Morgan 1972, 1982, 1993, 1997), there is no doubt that, at least in theory, waterside models do attempt to explain all of these elements by proposing a rather simple shift in adaptive pressure for moving through water and procuring food from aquatic habitats. In this regard, wading is just one of three modes of locomotion (swimming and diving being the other two) which may have had an effect on human evolution.

\section{References}

Abitbol, M. M. (1995). Lateral View of Australopithecus afarensis. Primitive Aspects of 
Bipedal Positional Behaviour in the Earliest Hominids. Journal of Human Evolution, 28, 211-229. https://doi.org/10.1006/jhev.1995.1017

Ashton, E. H., Flinn, R. M., Moore, W. J., Oxnard, C. E., \& Spence, T. F. (1981). Further Quantitative Studies of Form and Function in the Primate Pelvis with Special Reference to Australopithecus. Transactions of the Zoological Society of London, 36, 1-98. https://doi.org/10.1111/j.1096-3642.1981.tb00063.x

Bearder, S. (2000). Flood Brothers. BBC Wildlife, 18, 64-68.

Berge, C. (1984). Multivariate Analysis of the Pelvis for Hominids and Other Extant Primates: Implications for the Locomotion and Systematics of Different Species of Australopithecines. Journal of Human Evolution, 13, 555-562. https://doi.org/10.1016/S0047-2484(84)80027-5

Berge, C. (1994). How Did the Australopithecines Walk? A Biomechanical Study of the Hip and Thigh of Australopithecus afarensis. Journal of Human Evolution, 26, 259-273. https://doi.org/10.1006/jhev.1994.1016

Berge, C., \& Kazmierczak, J. B. (1986). Effects of Size and Locomotor Adaptations on the Hominid Pelvis: Evaluation of the Australopithecine Bipedality with a New Multivariate Method. Folia Primatologica, 46, 185-204. https://doi.org/10.1159/000156253

Bookstein, F. L. (1991). Morphological Tools for Landmark Data: Geometry and Biology. Cambridge: Cambridge University Press.

Breuer, T., Ndoundou-Hockemba, M., \& Fishlock, V. (2005). First Observation of Tool Use in Wild Gorillas. Public Library of Science (Biology), 3, e380.

Carey, T. S., \& Crompton, R. H. (2005). The Metabolic Costs of "Bent-Hip, Bent-Knee" Walking in Humans. Journal of Human Evolution, 48, 25-44. https://doi.org/10.1016/j.jhevol.2004.10.001

Claxton, A. G., Hammond, A. S., Romano, J., Oleinik, E., \& DeSilva, J. (2016). Virtual Reconstruction of the Australopithecus africanus Pelvis Sts 65 with Implications for Obstetrics and Locomotion. Journal of Human Evolution, 99, 10-24. https://doi.org/10.1016/j.jhevol.2016.06.001

Crompton, R. H., Yu, L., Wang, W. J., Gunther, M. M., \& Savage, R. (1998). The Mechanical Effectiveness of Erect and "Bent-Hip, Bent-Knee" Bipedal Walking in Australopithecus afarensis. Journal of Human Evolution, 35, 55-74. https://doi.org/10.1006/jhev.1998.0222

Ellis, D. V. (1993). Wetlands or Aquatic Ape? Availability of Food Resources. Nutrition and Health, 9, 205-217. https://doi.org/10.1177/026010609300900306

Franklin, D., Freedman, L., \& Milne, N. (2005). Three-Dimensional Technology for Linear Morphological Studies: A Re-Examination of Cranial Variation in Four Southern African Indigenous Populations. HOMO-Journal of Comparative Human Biology, 56, 17-34. https://doi.org/10.1016/j.jchb.2004.07.004

Hardy, A. (1960). Was Man More Aquatic in the Past? New Scientist, 7, 642-645.

Häusler, M. (1992). New Insights into the Locomotion of Australopithecus africanus Based on the Pelvis. Evolutionary Anthropology, 11, 53-57. https://doi.org/10.1002/evan.10056

Häusler, M., \& Berger, L. R. (2001). Stw 441/465: A New Fragmentary Ilium of a Small-Bodied Australopithecus africanus from Sterkfontein, South Africa. Journal of Human Evolution, 40, 411-417. https://doi.org/10.1006/jhev.2001.0465

Häusler, M., \& Schmid, P. (1995). Comparison of the Pelves of Sts 14 and AL 288-1: Implications for Birth and Sexual Dimorphism in Australopithecines. Journal of Human Evolution, 29, 363-383. https://doi.org/10.1006/jhev.1995.1063 
Hunt, K. D. (1994). The Evolution of Human Bipedality: Ecology and Functional Morphology. Journal of Human Evolution, 26, 183-202.

https://doi.org/10.1006/jhev.1994.1011

Hunt, K. D. (1996). The Postural Feeding Hypothesis: An Ecological Model for the Evolution of Bipedalism. South African Journal of Science, 92, 77-90.

Johanson, D. C., \& Edey, M. (1981). Lucy: The Beginnings of Humankind. New York: Simon \& Schuster.

Karlowski, U. (1996). The Conkouati Chimpanzee Refuge-A New Chance for Orphans. Gorilla Journal, 12, 20.

Kimbel, W. H., Johanson, D. C., \& Rak, Y. (1994). The First Skull and Other New Discoveries of Australopithecus afarensis at Hadar, Ethiopia. Nature, 368, 449-451. https://doi.org/10.1038/368449a0

Kingdon, J. (2003). Lowly Origins. Woodstock: Princeton University Press.

Kuliukas, A. V. (2001). Bipedal Wading in Hominoidea Past and Present. Masters Thesis, London: UCL.

Kuliukas, A. V. (2002). Wading for Food: The Driving Force of the Evolution of Bipedalism? Nutrition and Health, 16, 267-289. https://doi.org/10.1177/026010600201600402

Kuliukas, A. V. (2011). A Wading Component in the Origin of Hominin Bipedalism. In: M. Vaneechoutte, M. Verhaegen, \& A.V., Kuliukas, (Eds.), Was Man More Aquatic in the Past? Fifty Years after Alister Hardy: Waterside Hypothesis of Human Evolution. Basel: Bentham.

Kuliukas, A. V. (2013). Wading Hypotheses of the Origin of Human Bipedalism. Human Evolution, 28, 213-236.

Kuliukas, A. V. (2017). A Wading Component in the Origin of Hominin Bipedality? Ph.D. Thesis, Crawley: UWA.

Kuliukas, A. V., Milne, N., Fournier, P. A. (2009). The Relative Cost of Bent-Hip Bent-Knee Walking Is Reduced in Water. HOMO-Journal of Comparative Human Biology, 60, 479-488. https://doi.org/10.1016/j.jchb.2009.09.002

Latimer, B. (1991). Locomotor Adaptations in Australopithecus Afarensis: The Issue of Arboreality. In Y. Coppens, \& B. Senut (Eds.), Origine(s) de la bipedalie chez les hominides. Paris: CNRS.

Le Gros Clark, W. E. (1955). The OS Innominatum of the Recent Ponginae with Special Reference to That of the Australopithicinae. American Journal of Physical Anthropology, 13, 19-27. https://doi.org/10.1002/ajpa.1330130103

Lovejoy, C. O. (1979). A Reconstruction of the Pelves of AL 288 (Hadar Formation, Ethiopia). American Journal of Physical Anthropology, 50, 460.

Lovejoy, C. O. (1981). The Origin of Man. Science, 211, 341-350. https://doi.org/10.1126/science.211.4480.341

Lovejoy, C. O., \& Heiple, K. G. (1970). A Reconstruction of the Femur of Australopithecus africanus. American Journal of Physical Anthropology, 32, 33-40. https://doi.org/10.1002/ajpa.1330320105

Lovejoy, C. O., Heiple, K. G., \& Burstein, A. H. (1973). The Gait of Australopithecus. American Journal of Physical Anthropology, 38, 757-780. https://doi.org/10.1002/ajpa.1330380315

McHenry, H., \& Corruccini, R. S. (1975). Multivariate Analysis of Early Hominid Pelvic Bones. American Journal of Physical Anthropology, 43, 263-270.

https://doi.org/10.1002/ajpa.1330430212 
Morgan, E. (1972). The Descent of Woman. London: Souvenir Press.

Morgan, E. (1982). The Aquatic Ape. London: Souvenir Press.

Morgan, E. (1990). The Scars of Evolution. Oxford: Oxford University Press.

Morgan, E. (1993). Bipedalism. Nutrition and Health, 9, 193-204. https://doi.org/10.1177/026010609300900305

Morgan, E. (1997). The Aquatic Ape Hypothesis. London: Souvenir Press.

Myers-Thompson, J. A. (2002). Bonobos of the Lukuru. Wildlife Research Project. In C. Boesch, G. Hohman, \& L. F. Marchant (Eds.), Behavioural Diversity in Chimpanzees and Bonobos. Cambridge: Cambridge University Press.

Niemitz, C. (2002). A Theory on the Evolution of the Habitual Orthograde Human Bipedalism-The "Amphibische Generalisten Theorie". Anthropologischer Anzeiger, 60, 3-66.

Niemitz, C. (2004). Das Geheimnis des Aufrechten Gangs: Unsere Evolution Verlief Anders. Munich: Beck, C. H.

Niemitz, C. (2007). Labil und langsam. Naturwissenschaftliche Rundschau, 60, 71-77.

Niemitz, C. (2010). The Evolution of the Upright Posture and Gait-A Review and a New Synthesis. Naturwissenschaften, 97, 241-263. https://doi.org/10.1007/s00114-009-0637-3

Oxnard, C. E. (1975). The Place of the Australopithecines in Human Evolution: Grounds for Doubt? Nature, 258, 389-395. https://doi.org/10.1038/258389a0

Oxnard, C. E. (1979). The Relationship of Australopithecus and Homo: Another View. Journal of Human Evolution, 8, 427-432. https://doi.org/10.1016/0047-2484(79)90080-0

Oxnard, C. E., \& Hoyland-Wilkes, C. (1994). Hominid Bipedalism or Bipedalisms? The Pelvic Evidence. Perspectives in Human Biology, 1994, 13-34.

Sellers, W. I., Dennis, L. A., Wang, W. J., \& Crompton, R. H. (2004). Evaluating Alternative Gait Strategies Using Evolutionary Robotics. Journal of Anatomy, 204, 331-343. https://doi.org/10.1111/j.0021-8782.2004.00294.x

Stern Jr., J. T., \& Susman, R. L. (1983). The Locomotor Anatomy of Australopithecus afarensis. American Journal of Physical Anthropology, 60, 279-317. https://doi.org/10.1002/ajpa.1330600302

Tague, R. G., \& Lovejoy, C. O. (1986). The Obstetric Pelvis of A.L. 288-1 (Lucy). Journal of Human Evolution, 15, 237-255. https://doi.org/10.1016/S0047-2484(86)80052-5

Tutin, C. E. G., Ancrenaz, M., Vacher-Vallas, M., Vidal, C., Bruford, M. W., Jamart, A., Paredes, J., \& Goossens, B. (2001). Conservation Biology Framework of the Release of Wild-Born Orphaned Chimpanzees into the Conkouati Reserve, Congo. Conservation Biology, 15, 1247-1257.

Verhaegen, M., Puech, P. F., \& Munro, S. (2002). Aquarboreal Ancestors? Trends in Ecology and Evolution, 17, 212-217. https://doi.org/10.1016/S0169-5347(02)02490-4

Vignaud, P., Duranger, P., Mackaye, H., Likius, A., Blondel, C., Boisserie, J., de Bonis, L., Eisenmann, V., Etienne, M., Geraads, D., Guy, F., Lehmann, T., Lihoreau, F., Lopez-Martinez, N., Mourer-Chauvire, C., Otero, O., Rage, J., Schuster, M., Viriot, L., Zazzo, A., \& Brunet, M. (2002). Geology and Palaeontology of the Upper Miocene Toros-Menalla Hominid Locality, Chad. Nature, 418, 152-155. https://doi.org/10.1038/nature00880

Westenhöfer, M. (1942). Der Eigenweg des Menschen. Berlin: Mannstaedt \& Co.

Wrangham, R., Cheney, D. L., Seyfarth, R., \& Sarmiento, E. E. (2009). Shallow-Water 
Habitats as Sources of Fall-back Foods for Hominins. American Journal of Physical Anthropology, 140, 630-642. https://doi.org/10.1002/ajpa.21122

Zuckerman, S., Ashton, E. H., Flinn, R. M., Oxnard, C. E., \& Spence, T. F. (1973). Some Locomotor Features of the Pelvic Girdle in Primates. Symposia of the Zoological Society of London, 33, 71-165. 


\section{A1. Appendices/Supplementary Materials}

\subsection{General 3D GM Study Methods}

\subsubsection{Landmarks Types}

A comprehensive series of landmarks was determined for the pelvis and femur for several species of Hominoidea (extant or extinct).

Two broad categories of landmarks types were obtained, the first based on the taxonomy of landmarks as defined by Bookstein (1991) and Marcus et al. 1996.

1) Easily-recognisable "point" landmarks. These are the types that have been traditionally used in morphometric studies of the skeleton in the past both classically, where direct, instrumentally derived, measurements have been taken between two points, and more recently in studies such as this, where individual 3D co-ordinates have been captured, from which the traditional measures can still be derived.

2) Semi-landmarks. Several series of semi-landmarks were included to provide a means of analysing linear shapes e.g. the linea aspera, of the femur and the iliac crest, the brim inlet and outlet of the pelvis.

\subsubsection{Pelvic Landmark Schema}

Table 1 lists the point landmarks used on the pelvis.

These landmarks are illustrated graphically in the Figures 1-3, Table 2, Table 3.

\subsubsection{Species Studied}

\section{1) Species list}

This study does not cover the full range of primates covered by some earlier studies (e.g. ...), neither are the sample sizes used as great as in most of them, but it does cover all the major fossil hominids found before the discovery of $\mathrm{Ar}$ dipithecus ramidus (Lovejoy et al. 2009). Due to the relatively incomplete nature of that fossil specimen it was decided to omit Ardipithecus from this study (Table 4).

\subsubsection{Data Capture}

The 3D landmarks were captured and written directly to Microsoft Excel data files using a Microscribe Digitiser (Immersion Corporation, San Jose, CA), and associated software. As shown in Figure 4. The sample and the digitiser remained fixed during the recording of a set of landmarks. Where, occasionally, the sample or digitiser needed to be moved (perhaps to get access to landmarks that were hidden from the first orientation), the two sets of $3 \mathrm{D}$ points were re-aligned digitally through software using four reference landmarks common to both sets.

The digitiser has a heavy base and the samples were fixed to the work surface either with a clamp or adhesive material.

\subsubsection{Pre-Processing Techniques}

A number of generated landmarks and landmark processing methods were used 
Table 1. Pelvic landmark schema (Hip bone).

\begin{tabular}{|c|c|c|c|}
\hline LM & Type & Code & Description \\
\hline \multicolumn{4}{|c|}{ Left Side Hip bone Landmarks (right side has 46 corresponding symmetrical ones with "R" suffix) } \\
\hline 1 & 1 & AcCentL & Deepest \& most central point in the acetabulum and point of fusion of ischium, ilium and pubis. \\
\hline 2 & 3 & AcRimIschL & $\begin{array}{l}\text { Rim of the acetabulum at the intersection of the axis of the ischium-as defined by the linear } \\
\text { buttress emanating from it in the direction of the Ischial tuberosity generally. }\end{array}$ \\
\hline 3 & 3 & AcRimILL & Rim of the acetabulum at the intersection of the cranio-lateral border of the iliac blade. \\
\hline 4 & 3 & AcRimPubL & Point on the rim of the acetabulum intersected by the axis of the pubic bone. \\
\hline 5 & 3 & AcRimPubOpL & Point on the opposite rim of the acetabulum intersected by the axis of the pubic bone. \\
\hline 6 & 3 & AcNchVntL & Ventral margin of acetabular notch (on rim.) \\
\hline 7 & 3 & AcNchDrsL & Dorsal margin of acetabular notch (on rim.) \\
\hline 8 & 3 & AcArtSL & $\begin{array}{l}\text { Most central limit of the inner acetabular articular surface on the line perpendicular to } \\
{[\text { AcNchDrsL] and }[A c N c h V n t L] .}\end{array}$ \\
\hline 9 & 1 & oRecFemRHL & Origin of the rectus femoris reflected head on groove just above acetabulum. \\
\hline 10 & 1 & AIISL & Anterior inferior iliac spine, most caudal (i.e. furthest along the ilium from the sacrum) point. \\
\hline 11 & 1 & ASISL & $\begin{array}{l}\text { The most caudal point on the anterior superior iliac spine which, when observed } \\
\text { perpendicularly to the iliac plane, has the greatest curvature. }\end{array}$ \\
\hline 12 & 3 & oTFLL & Mid point of IL-TUB-GL and ASIS (origin of Tensor Fascia Latae.) \\
\hline 13 & 1 & IlTubExL & Iliac Tubercle, external. The point on the gluteal margin of thickest width of iliac crest cranial to ASIS. \\
\hline 14 & 1 & IlTubInL & $\begin{array}{l}\text { Iliac Tubercle, internal. The point on the interior surface on the crest of thickest width of iliac } \\
\text { crest cranial to ASIS. }\end{array}$ \\
\hline 15 & 3 & CranIlL & Furthest (most cranial) point on the Iliac crest from AcCentL. \\
\hline 16 & 1 & CrstSacIntL & Intersection of the iliac crest (interior margin) with the buttress leading to the sacrum. \\
\hline 17 & 1 & CrstSacExtL & Intersection of the iliac crest (exterior margin) with the buttress leading to the sacrum. \\
\hline 18 & 3 & DorsILL & The most dorsal point on the iliac crest. (This lies close to the posterior superior iliac spine). \\
\hline 19 & 1 & PSISL & Posterior superior iliac spine. \\
\hline 20 & 1 & oES-LatL & $\begin{array}{l}\text { Most lateral point on the erector-spinae origin surface at the midpoint of PSIS and oPirif-GScNL. } \\
\text { Indicates strength of erector spinae. }\end{array}$ \\
\hline 21 & 1 & oES-MedL & $\begin{array}{l}\text { Most medial point on the erector-spinae origin surface at the midpoint of PSIS and oPirif-GScNL. } \\
\text { Indicates strength of erector spinae. }\end{array}$ \\
\hline 22 & 1 & oPirif-GScNL & $\begin{array}{l}\text { Medial end of the greater sciatic notch, where smooth curvature ends (also in humans acts a } \\
\text { partial origin to piriformis). }\end{array}$ \\
\hline 23 & 1 & SIJ-CranL & Junction of cranial limit of sacro-iliac joint and iliac crest. \\
\hline 24 & 1 & SIJ-BrimL & Junction of the auricular surface with the pelvic brim. \\
\hline 25 & 1 & SIJ-CaudL & Caudal limit of auricular surface close to Posterior inferior iliac spine PIIS. \\
\hline 26 & 4 & LatInletL & $\begin{array}{l}\text { Most lateral point on the iliopectineal line on hip bone (forms transverse diameter with opposite) } \\
\text { On fully articulated only. May calculate from disarticulated by taking a trace of points on the inlet. }\end{array}$ \\
\hline 27 & 4 & MidInletL & Mid point of PUBSA to SIJ-PI on the pelvic. \\
\hline 28 & 1 & IPEL & ILIO-pectineal eminence. \\
\hline 29 & 4 & OBLIQUEL & $\begin{array}{l}\text { Oblique diameter with opposite SIJ-PI) on the margin of the brim alongside IPE } \\
\text { (from: Midwifery-based True pelvis dimensions). }\end{array}$ \\
\hline 30 & 4 & oPectL & Half way point between IPEL and PubTubL (origin of Pectineus). \\
\hline 31 & 1 & PubTubL & Most ventral corner of caudal surface of the pubic tubercle (origin of Adductor Longus). \\
\hline 32 & 1 & PubCranL & Most cranial point, at maximum height, of the pubic symphysis. \\
\hline 33 & 1 & PubCaudL & Most caudal point on pubic symphysis. \\
\hline
\end{tabular}




\section{Continued}

\begin{tabular}{|c|c|c|c|}
\hline 34 & 1 & PubDorsL & Closest point on the pubis to the sacral promontory indicating maximum $\mathrm{D}-\mathrm{V}$ extension of pelvic inlet. \\
\hline 35 & 3 & SciNotL & $\begin{array}{l}\text { That point situated on the margin of the greater sciatic notch which is most distant from the } \\
\text { PSISL-IschSpL axis. }\end{array}$ \\
\hline 36 & 1 & IschSpL & Point of greatest curvature on the ischial spine. \\
\hline 37 & 3 & oObtIntL & The rim of the lesser sciatic notch (origin of Obturator internus and Gemelli). \\
\hline 38 & 1 & IT-CranL & Most cranial limit of ischial tuberosity. \\
\hline 39 & 1 & IT-CaudL & Most caudal point on the ischial tuberosity. \\
\hline 40 & 1 & IT-ExtL & Most lateral (external) point of ischial tuberosity. \\
\hline 41 & 1 & IT-IntL & Most medial (internal) point of ischial tuberosity. \\
\hline 42 & 4 & IT-CentL & Central point of the ischial tuberosity (intersection of IT-ExtL + IT-IntL, and IT-CranL + IT-CaudL). \\
\hline 43 & 3 & OFI-AcL & Closest point on the interior rim of the obturator foramen to the mid-point of [AcNchVntL] and [AcNchDrsL]. \\
\hline 44 & 3 & OFI-PubL & The point on the obturator foramen border closest to the cranial margin of pubic symphysis. \\
\hline 45 & 3 & OFI-IschL & The point on the obturator foramen border closest to [IT-CentL]. \\
\hline 46 & 3 & OFI-RamL & The mid-point between OFI-PubL and OFI-IschL on the most caudal interior margin of obturator foramen. \\
\hline
\end{tabular}

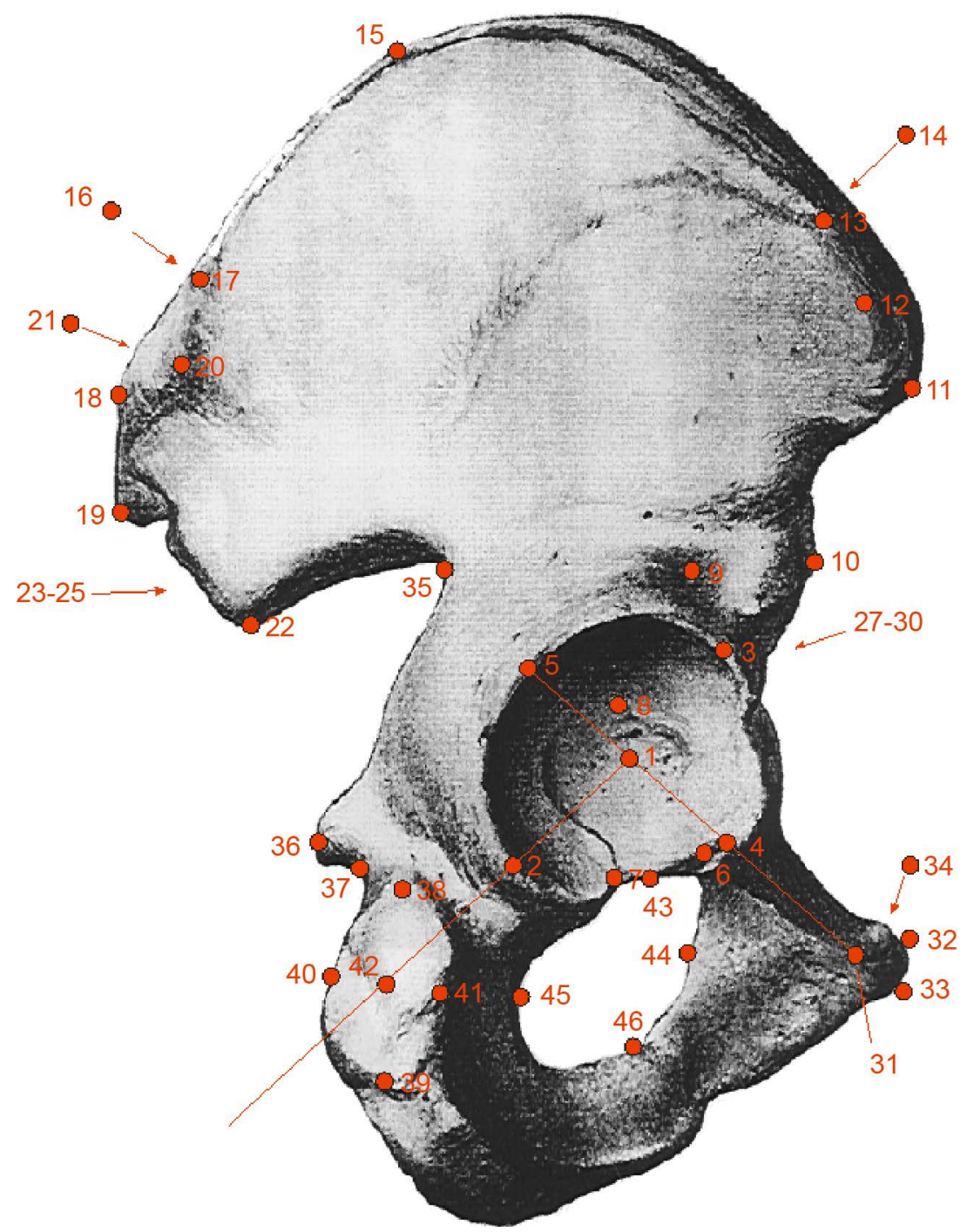

Figure 1. Landmarks of the Hip bone (lateral view). 


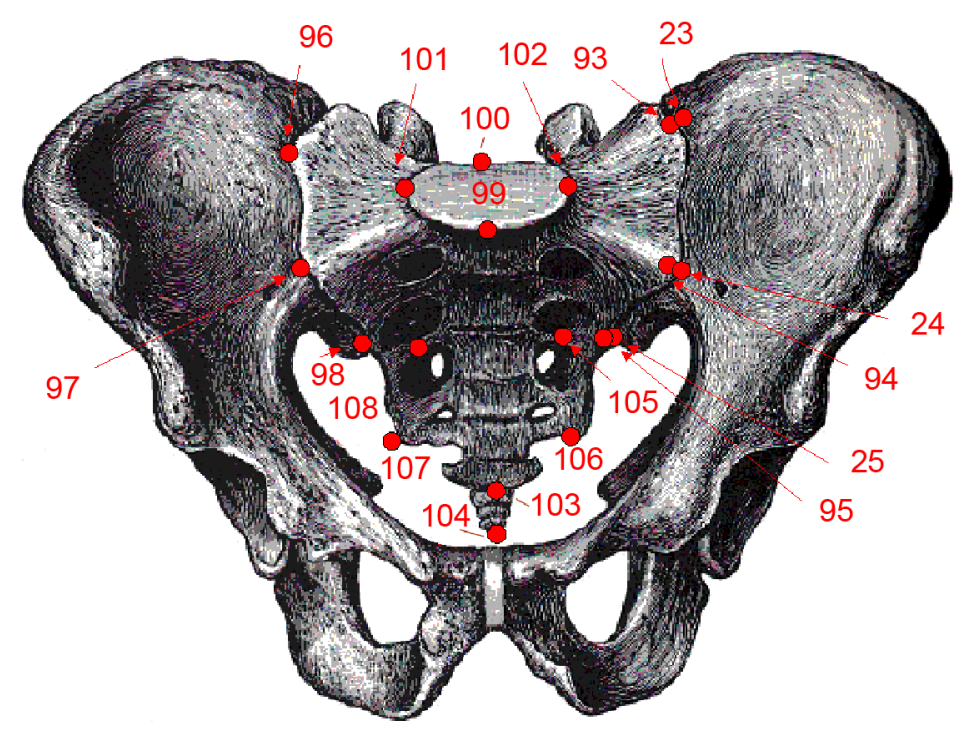

Figure 2. Landmarks of the Hip bone and Sacrum (Frontal View).

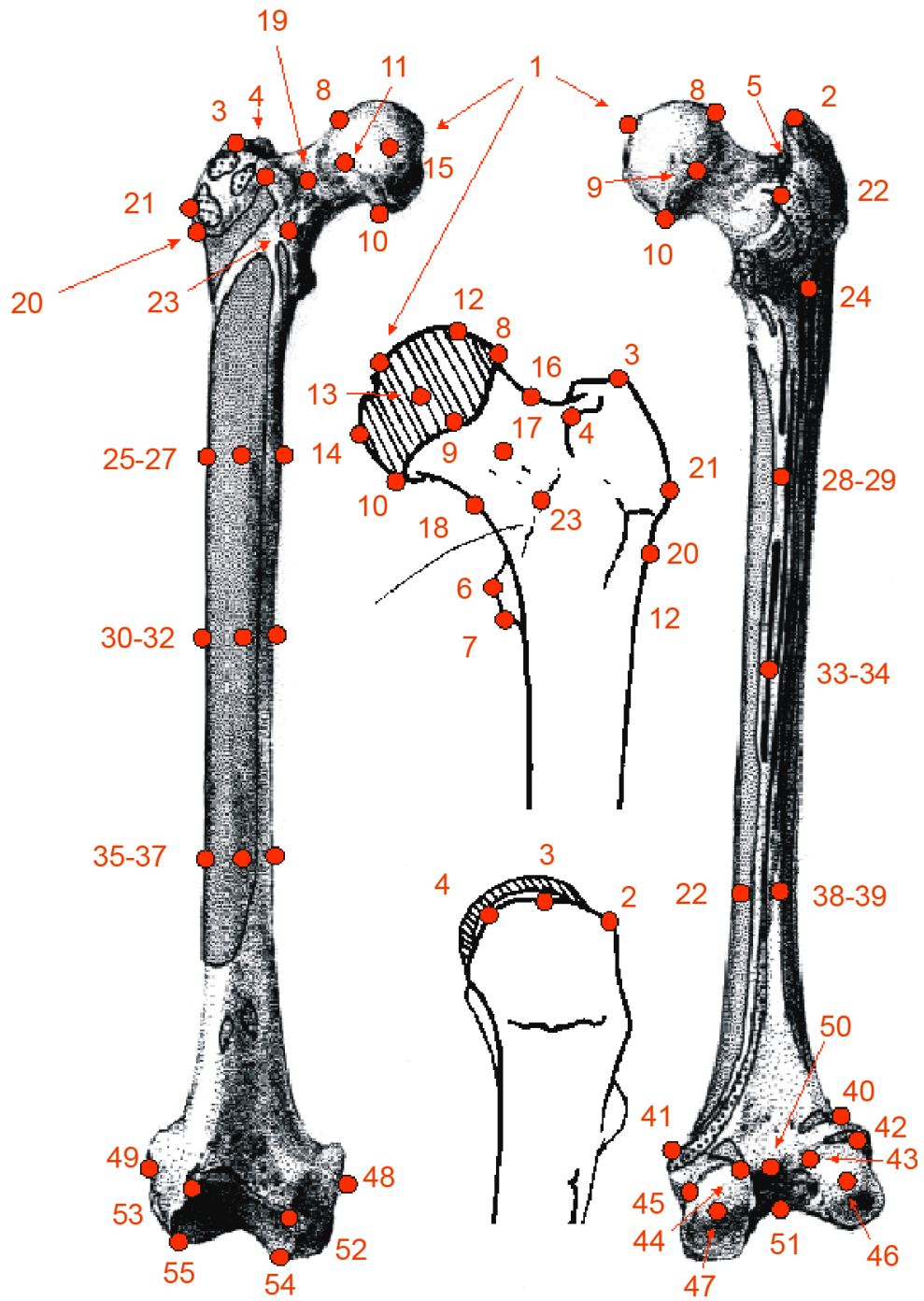

Figure 3. Landmarks of the femur. 
Table 2. Pelvic landmark schema (Sacrum).

\begin{tabular}{|c|c|c|c|}
\hline \multicolumn{4}{|r|}{ Landmarks on the Sacrum } \\
\hline 93 & 1 & sSIJ-CranL & Junction of cranial limit of sacro-iliac joint and iliac crest. \\
\hline 94 & 1 & sSIJ-BrimL & Junction of the auricular surface and the pelvic brim, left side. \\
\hline 95 & 1 & sSIJ-CaudL & Caudal limit of auricular surface. \\
\hline 96 & 1 & sSIJ-CranR & Opposite Junction of cranial limit of sacro-iliac joint and iliac crest. \\
\hline 97 & 1 & sSIJ-BrimR & Junction of the auricular surface and the pelvic brim, right side. \\
\hline 98 & 1 & sSIJ-CaudR & Opposite caudal limit of auricular surface. \\
\hline 99 & 1 & sVertProm & Sacral promontory in the plane of the pelvic inlet. Most cranio-ventral point on the sacral body. \\
\hline 100 & 1 & sVertDors & Most dorsal point on the sacral body. \\
\hline 101 & 1 & sVertLatR & Most lateral point on sacral vertebral joint right. \\
\hline 102 & 1 & sVertLatL & Most lateral point on sacral vertebral joint left. \\
\hline 103 & 1 & sSacTip & Most caudo-ventral tip of the sacrum. \\
\hline 104 & 1 & sCoxTip & Most caudal tip of the coccyx. \\
\hline 105 & 3 & oPirif-SACR & $\begin{array}{l}\text { Ventral surface of the right side sacrum between second and third foramen midway between midline } \\
\text { of foramen and lateral border. }\end{array}$ \\
\hline 106 & 1 & oGluMaxInfR & $\begin{array}{l}\text { Right infero-lateral angle, marking corner of sacrum (origin of coccygeus) where medial border of } \\
\text { sacrum begins to taper towards the coccyx (origin of Gluteus Maximus inferior fibers). }\end{array}$ \\
\hline 107 & 1 & oGluMaxInfL & $\begin{array}{l}\text { Left infero-lateral angle, marking corner of sacrum (origin of coccygeus) where medial border of } \\
\text { sacrum begins to taper towards the coccyx (origin of Gluteus Maximus inferior fibers). }\end{array}$ \\
\hline 108 & 3 & oPirif-SACL & $\begin{array}{l}\text { Ventral surface of the left side sacrum between second and third foramen midway between midline } \\
\text { of foramen and lateral border (first sacral foramen is most cranial) (origin of Piriformis). }\end{array}$ \\
\hline
\end{tabular}

Table 3. Femoral landmark schema.

\begin{tabular}{cccl}
\hline LnkNo & LM Type & Landmark Name & \multicolumn{1}{c}{ "Point" Landmarks of the Femur } \\
\hline 1 & 2 & FemHeadCtr & The centre of the femoral head in line with the axis of the femoral neck \\
2 & 1 & GtTrochPost & Most ant.-posterior point on the greater trochanter \\
3 & 2 & GtTrochAnt & $\begin{array}{l}\text { Most anterior-superior point of greater trochanter (corresponds with insertion of } \\
\text { Gluteus minimus }\end{array}$ \\
4 & 1 & GtTrochAnt & Most superior point on the greater trochanter \\
5 & 1 & TrochFossa & Deepest point in the trochanteric fossa \\
6 & 1 & LesTrochSup & Most superior point on the lesser trochanter \\
7 & 1 & LesTrochInf & Most inferior point on the lesser trochanter \\
8 & 2 & FemHeadRimSup & Most superior point on the rim of the femoral head \\
9 & 2 & FemHeadRimPost & Most posterior point on the rim of the femoral head \\
10 & 2 & FemHeadRimInf & Most inferior point on the rim of the femoral head \\
11 & 2 & FemHeadRimAnt & Most anterior point on the rim of the femoral head \\
12 & 2 & FemHeadMaxSup & Most superior point on the head at maximum distance from the femoral head axis \\
13 & 2 & FemHeadMaxPost & Most posterior point on the head at maximum distance from the femoral head axis \\
14 & 2 & FemHeadMaxInf & Most inferior point on the head at maximum distance from the femoral head axis \\
15 & 2 & FemHeadMaxAnt & Most ant. point on the head at maximum distance from the femoral head axis \\
16 & 2 & FemNeckSup & Most sup. point on the mid-point of the femoral neck \\
\hline & & &
\end{tabular}




\section{Continued}

\begin{tabular}{|c|c|c|c|}
\hline 17 & 2 & FemNeckPost & Most post. point on the mid-point of the femoral neck \\
\hline 18 & 2 & FemNeckInf & Most inf. point on the mid-point of the femoral neck \\
\hline 19 & 2 & FemNeckAnt & Most anterior point on the mid-point of the femoral neck-point of attachment of ilio-femoral ligament \\
\hline 20 & 2 & FemAxis & Estimated point on the lateral extremity of the axis of the femoral neck \\
\hline 21 & 2 & TrochLat & Most lateral point on the greater trochanter \\
\hline 22 & 2 & QuadTub & Quadrate tubercle-most medial point \\
\hline 23 & 2 & AntIntTrochLine & Most anterior point on the inter-trochanteric line, borders the femoral neck \\
\hline 24 & 2 & ThrdTroch & 3rd Trochanter or most sp. point on gluteal ridge \\
\hline 25 & 2 & Shaft1Med & Medial-most point quarter of the way down the shaft \\
\hline 26 & 2 & Shaft1Lat & Lateral-most point quarter of the way down the shaft \\
\hline 27 & 2 & Shaft1Ant & Anterior-most point $1 / 4$ way down the shaft \\
\hline 28 & 2 & LinAsp1Med & Medial-most point on the linea aspera, quarter of the way down the shaft \\
\hline 29 & 2 & LinAsp1Lat & Lateral-most (if any) point on the linea aspera, quarter of the way down the shaft \\
\hline 30 & 2 & Shaft2Med & Medial-most point quarter of the way down the shaft \\
\hline 31 & 2 & Shaft2Lat & Lateral-most point quarter of the way down the shaft \\
\hline 32 & 2 & Shaft2Ant & Anterior-most point $1 / 4$ of the way down the shaft \\
\hline 33 & 2 & LinAsp2Med & Medial pt on the linea aspera on mid pt of the shaft \\
\hline 34 & 2 & LinAsp2Lat & Lateral-most point on the linea aspera, on mid point of the shaft \\
\hline 35 & 2 & Shaft3Med & Medial-most point on the shaft $3 / 4$ of the way down ${ }^{1}$ \\
\hline 36 & 2 & Shaft3Lat & Lateral-most point on the shaft $3 / 4$ of the way down ${ }^{1}$ \\
\hline 37 & 2 & Shaft3Ant & Anterior-most point on the shaft $3 / 4$ of the way down \\
\hline 38 & 2 & LinAsp3Med & Point three quarters of the way down the shaft on the medial side on the medial supracondylar line \\
\hline 39 & 2 & LinAsp3Lat & Point three quarters of the way down the shaft on the lateral side on the lateral supracondylar line \\
\hline 40 & 2 & LatCondyle & Most lateral inferior point on the popleatal surface (opposite adductor tubercle) \\
\hline 41 & 2 & MedAddTub & Adductor tubercle \\
\hline 42 & 2 & LatLatCond & Most superior-lateral point on the lateral condyle \\
\hline 43 & 2 & LatMedCond & Most superior-medial point on the lateral condyle \\
\hline 44 & 2 & MedLatCond & Most superior-medial point on the lateral condyle \\
\hline 45 & 2 & MedMedCond & Most superior-medial point on the medial condyle \\
\hline 46 & 2 & MedPostCond & Most posterior point on the medial condyle \\
\hline 47 & 2 & LatPostCond & Most posterior point on the lateral condyle \\
\hline 48 & 2 & MedEpiCond & Medial epicondyle \\
\hline 49 & 2 & LatEpiCond & Lateral epicondyle \\
\hline 50 & 2 & IntCondNotSup & Intercondylar notch superior point \\
\hline 51 & 2 & IntCondNotInf & Intercondylar notch inferior point \\
\hline 52 & 2 & AntMedCondyle & Most anterior-medial point on articular surface of the patella surface \\
\hline 53 & 2 & AntLatCondyle & Most anterior-lateral point on articular surface of the lateral condyle \\
\hline 54 & 2 & DistMedCondyle & Most distal point on the medial condyle ${ }^{1}$ \\
\hline 55 & 2 & DistLatCondyle & Most distal point on the lateral condyle ${ }^{1}$ \\
\hline 56 & 2 & Fovea & The centre of the fovea capitis \\
\hline
\end{tabular}


Table 4. Species studied.

\begin{tabular}{|c|c|c|c|}
\hline \multirow[b]{2}{*}{ Phylogenetic Grouping } & \multicolumn{3}{|l|}{ Species Studied } \\
\hline & Latin Name & Code & $\mathrm{N}$ \\
\hline 1 & Lemur catta & LEC & 1 \\
\hline 2 & Ateles geoffroyi & ATG & 1 \\
\hline 3.01.1.1 & Cercopithecus aethiops & CEA & 2 \\
\hline 3.01 .2 & Mandrillus sphinx & MSL & 1 \\
\hline 3.01 .3 & Theropithecus gelada & TGL & 1 \\
\hline 3.01 .4 & Nasalis larvatus & NSL & 2 \\
\hline 3.02 .1 & Hylobates syndactylus & HYL & 4 \\
\hline 3.02 .2 & Hylobates agilis & HYL & 2 \\
\hline 3.02 .3 & Hylobates lar & HYL & 1 \\
\hline 3.02 .4 & Hylobates moloch & HYL & 5 \\
\hline 3.02 .5 & Hylobates muelleri & HYL & 3 \\
\hline 3.03 .1 & Pongo abelii & PPA & 5 \\
\hline 3.03 .2 & Pongo pygmaeus & PPY & 11 \\
\hline 3.04 .1 & Gorilla gorillagorilla & GGO & 17 \\
\hline 3.04 .2 & Gorilla gorilla graueri & GGB & 10 \\
\hline 3.05 & Pan paniscus & PNP & 19 \\
\hline 3.06 & Pan troglodytes & PNT & 16 \\
\hline $3.07 \mathrm{X}$ & Australopithecus afarensis (AL 288-1) & AFA & 1 \\
\hline $3.08 \mathrm{X}$ & Australopithecus africanus (STS 14) & AFR & 1 \\
\hline $3.09 \mathrm{X}$ & Homo erectus ( WT 15000) & HER & 1 \\
\hline 3.10 & Homo sapiens sapiens & HSS & 32 \\
\hline
\end{tabular}

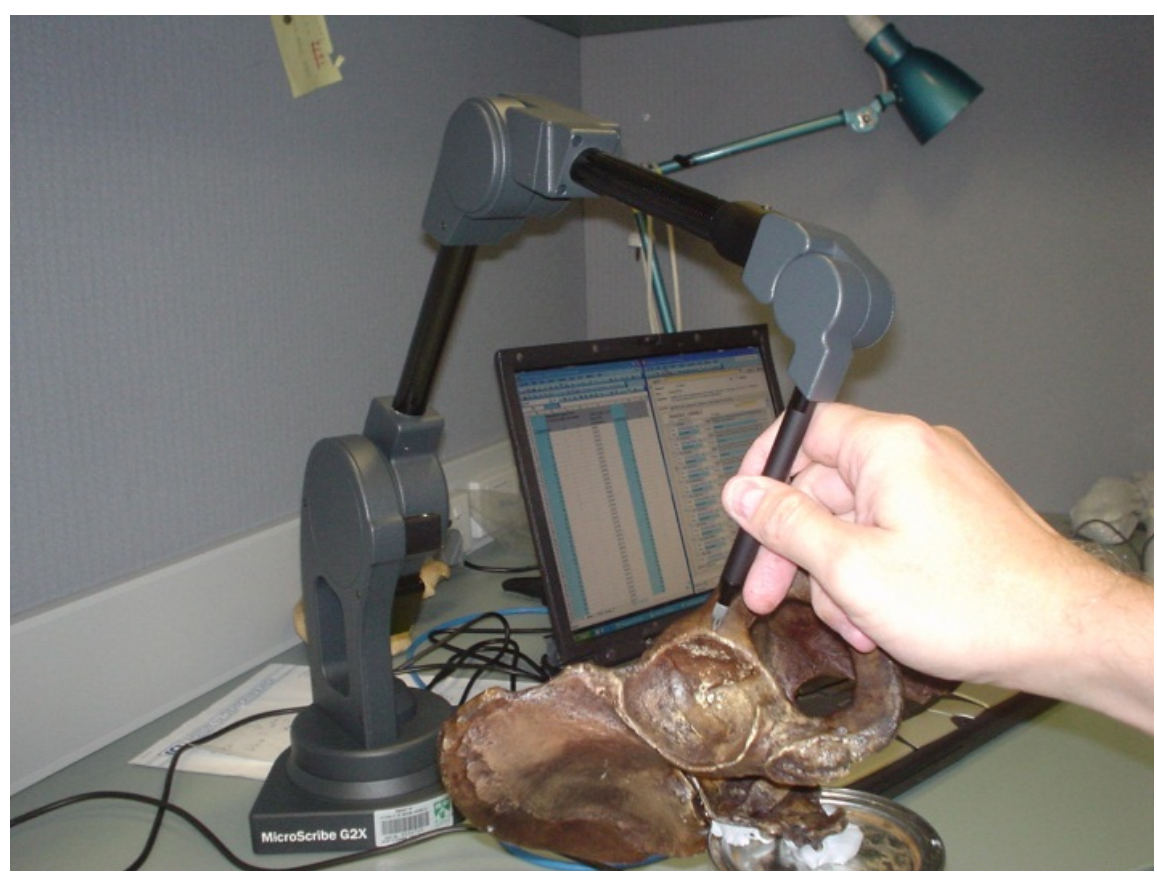

Figure 4. Digitising a model pelvis. 
in this study through software written by the author (programmable database, MorphDb—see section later.)

1) Generated landmarks on the hip

In order to analyse muscle Lever Arms, two generated landmarks were derived from the landmark schemas described above. One estimates the centre of rotation of the acetabulum and one estimates a hypothetical insertion point on the femur (at the greater trochanter). The following describes the method used to derive these landmarks.

2) Generated landmarks

A number of generated landmarks were derived from those listed in Table 5.

\subsection{MorphDB Relational Database}

\subsubsection{Introduction}

Throughout the study, hundreds of specimen of skeletal samples were digitised in multiple ways, generating over a million 3D co-ordinates. When collecting large quantities of digitised data, one soon accumulates many data files which require careful storage and organisation. Therefore it was decided that such a vast amount of data should be stored in a fully functional, programmable relational database.

As the database was almost exclusively for personal use, Microsoft Access was chosen, rather than a "higher end" solution, such as Oracle, SQL Server or MySQL. Microsoft Access is a popular (SQL-based) relational database with sophisticated development features such as a rich windows forms event model and uses a relatively simple programming language, Visual Basic for Applications (VBA), but is simpler and more accessible to others than developing in Java or the Microsoft.Net framework.

Figure 5 shows the front screen of the database which gives an indication of the structure of the database entities, each of which can be searched individually.

The study required inter-landmark distances (ILDs), angles and other calculations to be performed on the captured data. Equally, when using shape analysis software, such as Morphologika, it is necessary to carefully prepare complex data files in order to analyse pre-defined sets of morphs, landmarks and attributes.

Table 5. Generated landmarks.

\begin{tabular}{ll}
\hline Landmark Name & \multicolumn{1}{c}{$\begin{array}{c}\text { Generated Landmarks } \\
\text { Description\& Method of Calculation }\end{array}$} \\
\hline CntRotAcetab & $\begin{array}{l}\text { Estimated centre of rotation of the femoral head. Calculated as the mean } \\
\text { point of the two pairs of opposite landmarks on the rim of the acetabulum } \\
\text { (landmark nos } 2 \& 3, \text { and } 4 \text { \& 5) }\end{array}$ \\
FemNeckExtended & $\begin{array}{l}\text { Estimated position of the greater trochanter, assuming a continuation line } \\
\text { from the centre of the acetabulum (landmark } 1) \text { through the calculated } \\
\text { centre of rotation of the femoral head to a point at a distance equal to the } \\
\text { length of the femoral neck of the associated femur for the individual. }\end{array}$ \\
\hline
\end{tabular}




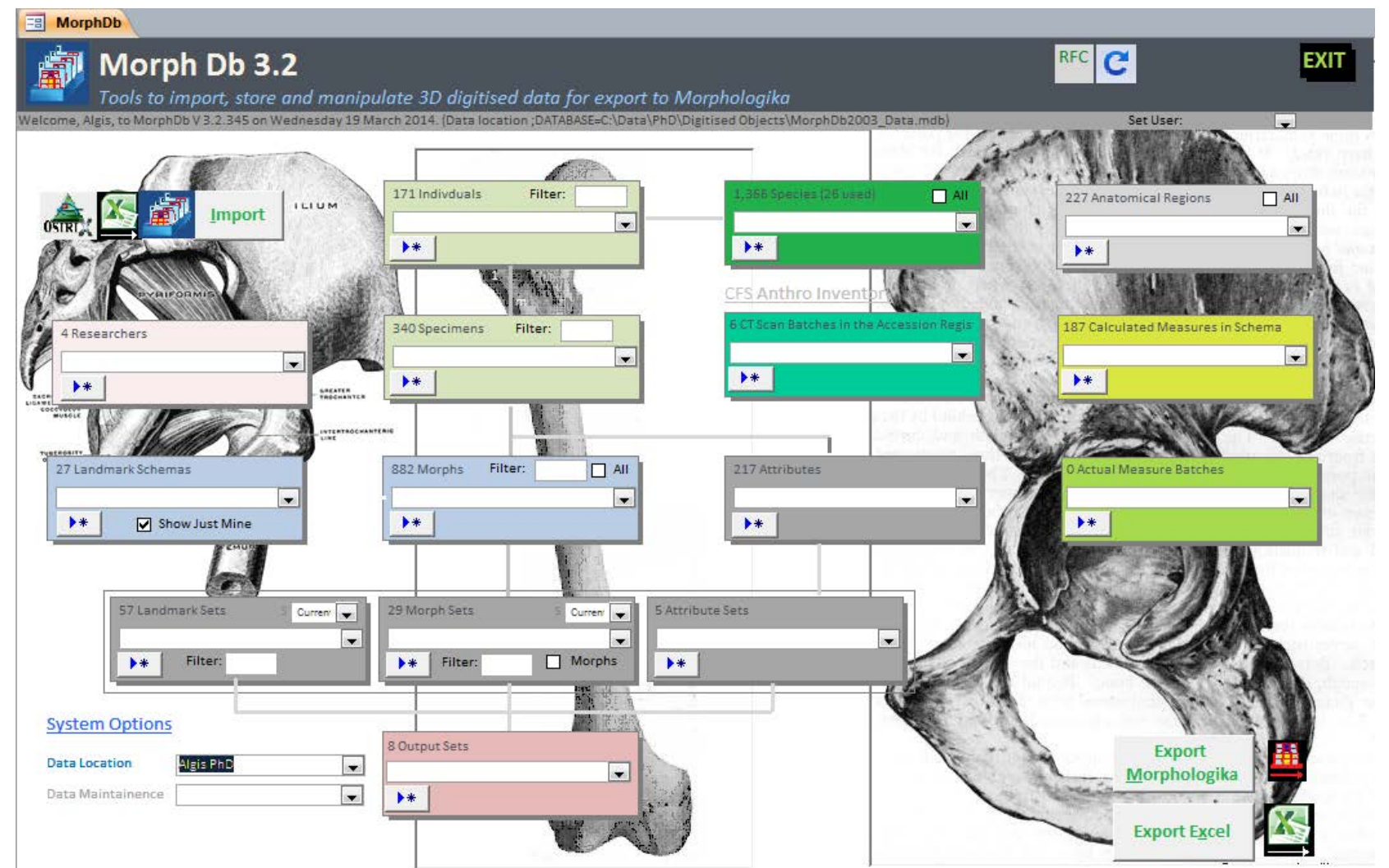

Figure 5. MorphDb-Home Page.

\subsubsection{General Function}

The database was designed to import 3D landmark data captured in the study from Comma separated variable (CSV), Excel and other file formats, store it in an easy-to-query relational database where the data can be manipulated and calculations performed before export data to other GM related software. An infinite number of sets of morphs, landmarks and attributes can be created and combined with each other before being analysed in Morphologika. Any combination of 3D landmarks can be used to calculate inter-landmark distances (ILDs), angle, and other metrics. This section briefly describes the database, which was designed by the author of his thesis.

\subsubsection{Functional Specification}

\section{1) Importing}

MorphDb can import digitised data captured either by a 3D digitiser (saved into Microsoft Excel format) or via Osirix DICOM reader (saved as Excel readable comma separated variable, CSV, files). Figure 6 shows the import dialog box and its various option.

As the data is imported into the database it is assigned to an existing species, individual, specimen, anatomical region, or such entities that are automatically created if they do not already exist.

MorphDb can import data in several pre-defined formats. For example, "region of interest" (ROI) files capture 3D landmarks from within the DICOM file 
rendering program, OsiriX. (See example from such a file in Figure 7.) Various processing steps are performed whilst importing some kinds of morph data, for example semi-landmarks can be generated from pre-defined sets of points.

Figure 8 shows an example of an Excel file generated by the 3D scanner...

Multiple files in a particular folder can be imported in one go to speed up the process.

Other file types (such as attribute files, landmark schemas and data from other MorphDb files) can also be imported.

2) Storage

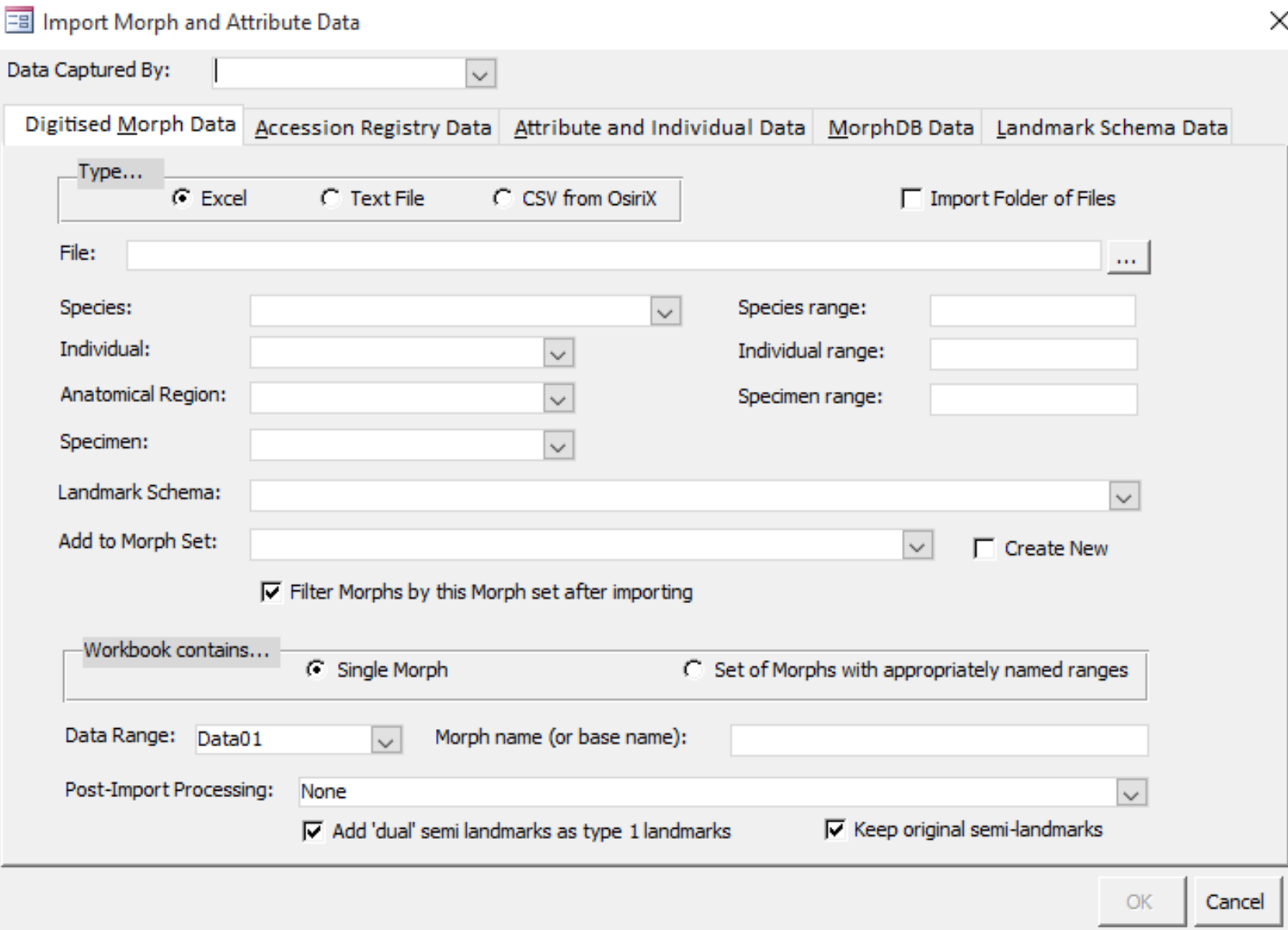

Figure 6. Import dialog box.

\begin{tabular}{|l|}
\hline ImageNo, Roi No,RoiMean, RoiMin, RoiMax, RoiT \\
$42,0,83.000000,83.000000,83.000000,83.00$ \\
$79,0,-404.000000,-404.000000,-404.000000$ \\
$96,0,-571.000000,-571.000000,-571.000000$ \\
$97,0,-97.000000,-97.000000,-97.000000,-9$ \\
$105,0,-7.000000,-7.000000,-7.000000,-7.0$ \\
$108,0,-560.000000,-560.000000,-560.00000$ \\
$115,0,-289.000000,-289.000000,-289.00000$ \\
$119,0,-376.000000,-376.000000,-376.00000$
\end{tabular}

Figure 7. Sample data from an OsiriX ROI file to be imported. 


\begin{tabular}{|c|c|c|c|c|c|}
\hline$\Delta$ & A & B & C & D & $E$ \\
\hline 1 & & \multirow{3}{*}{\multicolumn{3}{|c|}{$\begin{array}{l}\text { Description goes here } \\
\text { PpFBEL13201/nnomL }\end{array}$}} & \\
\hline 2 & & & & & $19 / 09 / 200519: 45$ \\
\hline 3 & & & & & Trace:001 \\
\hline 4 & LandmarkNo & $\mathrm{x}$ & $y$ & $z$ & Comments \\
\hline 5 & 1 & 253.3059 & -121.4456 & 217.9771 & point \\
\hline 6 & 2 & 269.2113 & -108.4206 & 237.1476 & point \\
\hline 7 & 3 & 262.9026 & -142.8218 & 224.4952 & point \\
\hline 8 & 4 & 275.2498 & -122.7945 & 212.6154 & point \\
\hline 9 & 5 & 246.6929 & -121.6271 & 241.5635 & point \\
\hline 10 & 6 & 267.506 & -130.8094 & 215.4698 & point \\
\hline 11 & 7 & 269.467 & -111.6381 & 224.4848 & point \\
\hline 12 & 8 & 248.0715 & -116.9613 & 229.5103 & point \\
\hline 13 & 9 & 243.2565 & -143.3679 & 224.8499 & point \\
\hline 14 & 10 & 245.365 & -159.7575 & 223.9785 & point \\
\hline
\end{tabular}

Figure 8. Sample data from Microscribe Digitiser file to be imported.

MorphDb was designed to store morphs in a normalised database structure, allowing them to be grouped and queried in very flexible ways. Millions of morphs can be stored, each containing potentially thousands of landmarks, and associated with hundreds of attributes.

Put simply, the database is organised hierarchically. Although defaulting to human anatomical data, any number of species can be imported for analysis. Individuals are stored along with any number of attributes, including sex and age. Each individual may have one or more associated specimens for a specific anatomical region (e.g. the pelvis or femur) of them. Each specimen can have associated one or more morphs. Each morph is linked to a landmark schema, which defines the landmarks to be captured, and a researcher. The database is multi-user and can contain and manipulate data from any number of researchers.

Each landmark schema may include any number of measures which define formulae to calculate inter-landmark distances, angles and other calculations from the landmarks. Once data has been imported into a morph, MorphDb will calculate all these defined measures automatically. Wireframe and polygon maps can also be stored in MorphDb for export to Morphologika.

The database is made of approximately 70 permanent tables in all, some of which are displayed in Figure 9.

\subsubsection{Manipulation}

\section{1) Set manipulation}

Morphs, landmarks and attributes can be grouped into many thousands of easily customisable sets (Figure 10 shows how individual landmarks are collected into an example set) which can be combined into almost infinite permutations. Any number of wireframes and polygons can be associated with each landmark schema and selected for output.

\section{2) ILD and other calculations}

MorphDb can store any number of pre-defined formulae in each landmark 


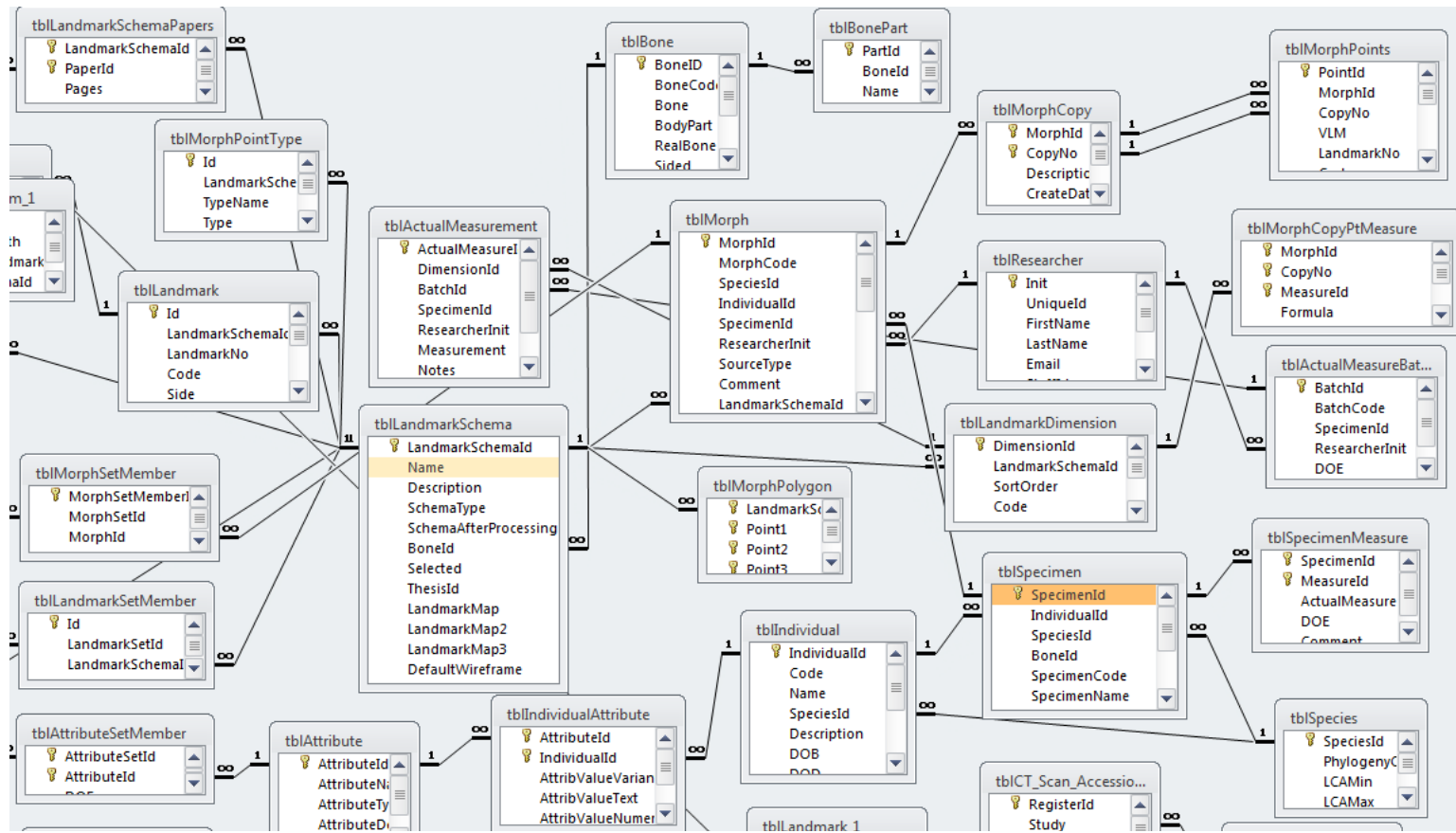

Figure 9. A Section of the Entity Relationship Diagram (ERD) of MorphDb.

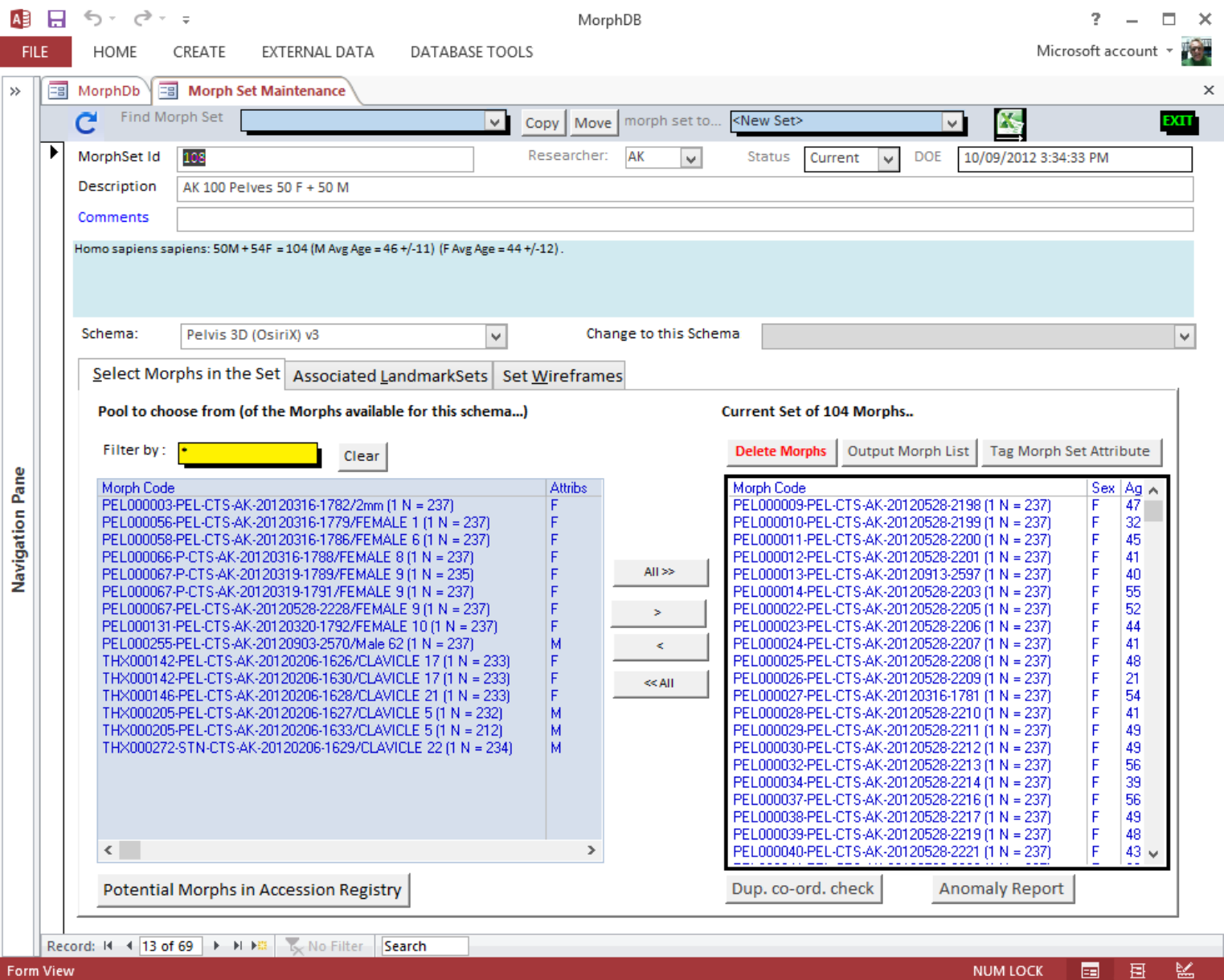

Figure 10. Morph set maintenance screen. 
schema which are then automatically calculated when data is imported and stored in a morph. Formulae may be simple inter-landmark distances, angles between three landmarks, subtenses-calculated distances between a landmark and a segment between two other landmarks, and many other types of calculation.

MorphDb therefore acts both as a pre-processor for complex 3D morphological analytical software, such as Morphologika, as well as a tool to calculate more traditional "linear" measures. These traditional measures can be validated and analysed for accuracy.

For example...

- An $\mathrm{n} \times \mathrm{m}$ precision analysis, where the researcher captures the same $\mathrm{n}$ specimen on $\mathrm{m}$ separate days. Here, standard estimates of accuracy are included, such as TEM, rTEM and coefficient of variability (Figure 11).

- A inter-researcher comparison, where the same specimen have been captured by two different researchers.

3) Export to morphologika

Along with calculating "traditional" linear measures, the major function of MorphDb is to output data to the $3 \mathrm{D}$ geometric morphometric analysis program Morphologika. Morphologika inputs a text file in a very specific format and MorphDb prepares these file precisely, but in flexible ways.

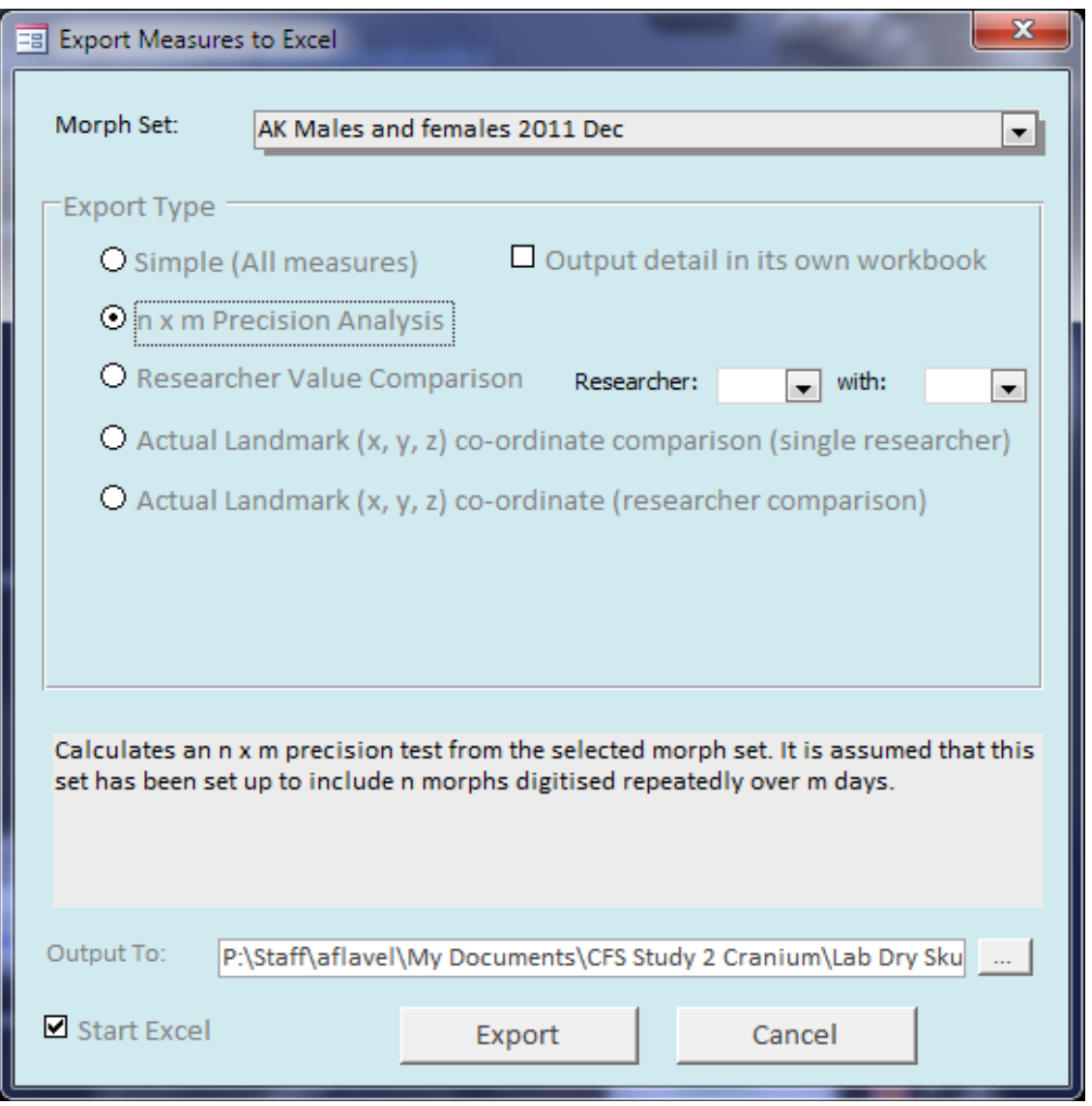

Figure 11. Some MorphDb export options. 
Basically, MorphDb combines sets of morphs, landmarks and attributes into a single Morphologika file. It also will generate a wireframe and/or 3D polygons to render the shapes in 3D (Figure 12).

An example target Morphologika file is shown in Figure 13.

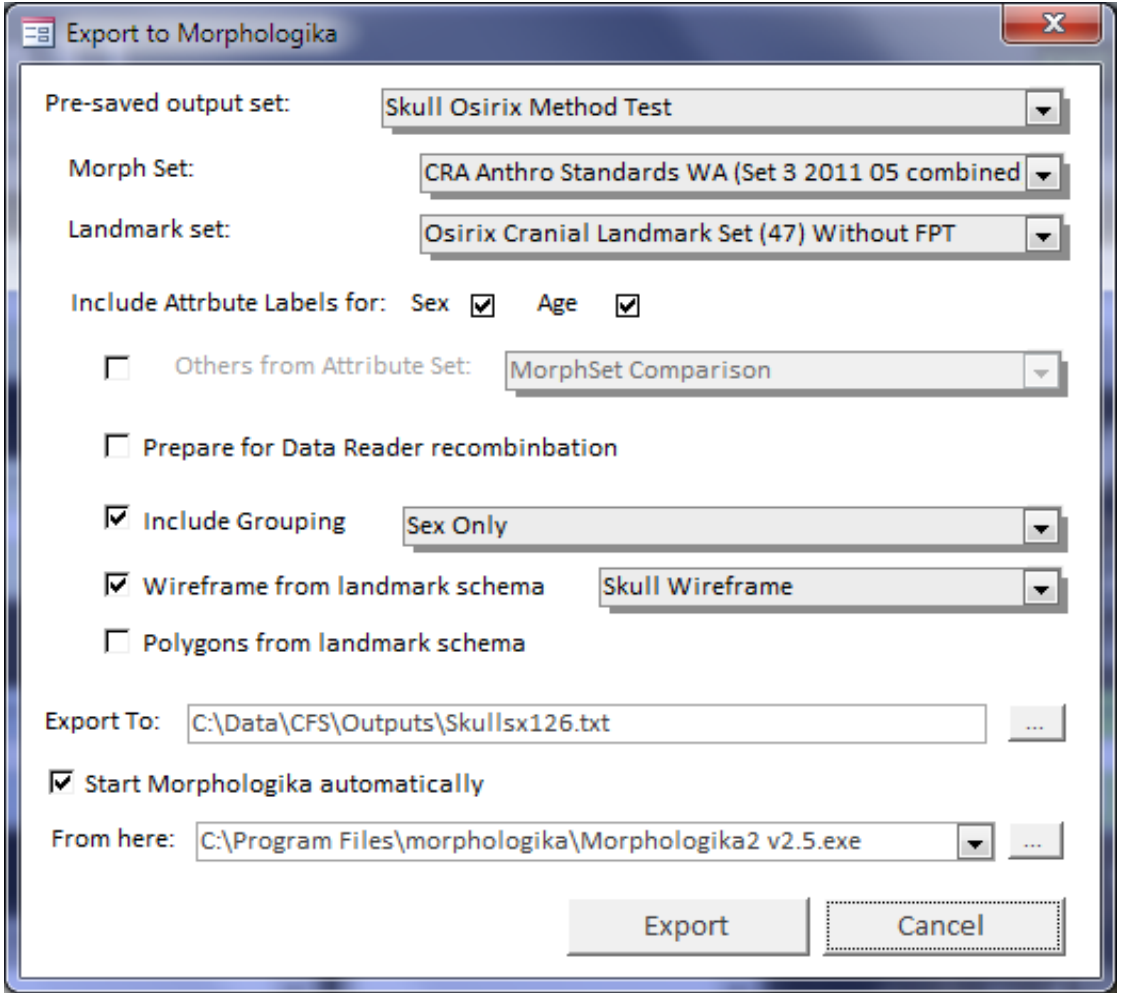

Figure 12. Exporting data to morphologika.

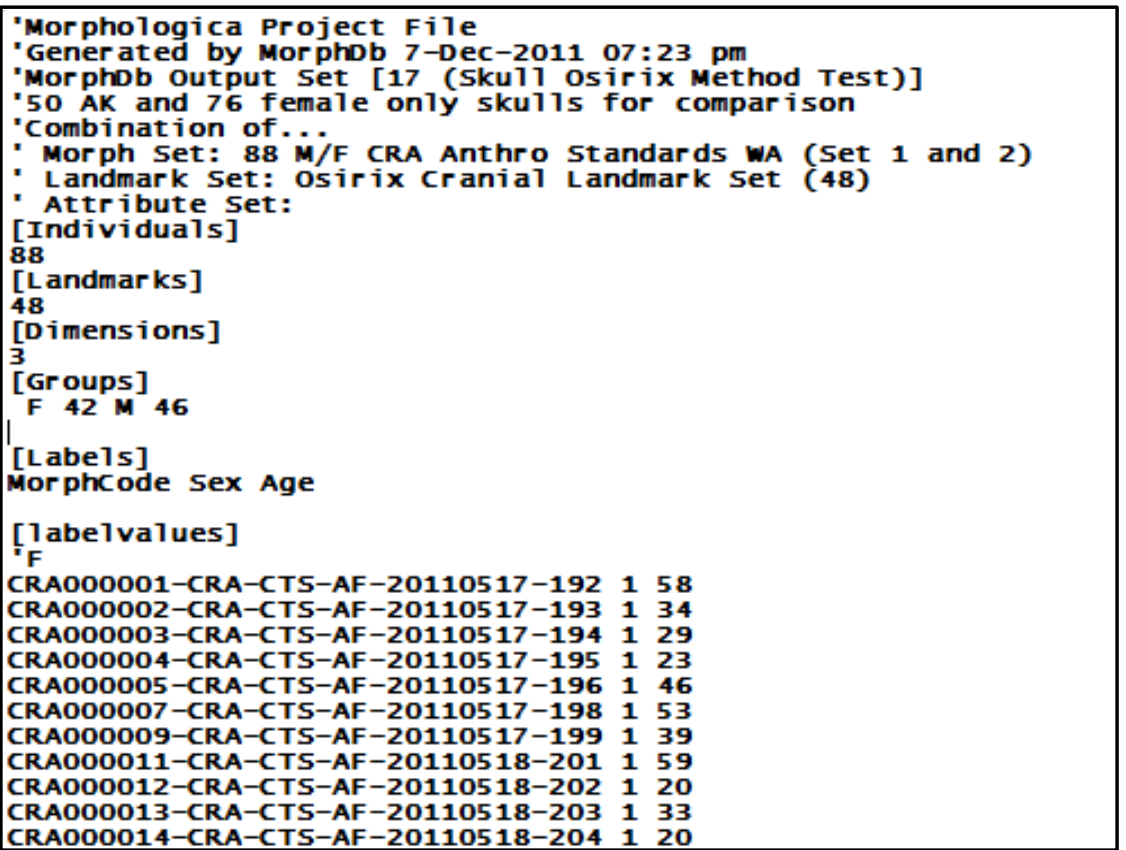

Figure 13. Example of a morphologika file. 


\section{4) Filtering and grouping by taxa}

Before the data were exported into Morphologika, they were filtered, allowing any combination of landmarks to be analysed, and sorted and grouped according to taxa (usually by sex/species permutations). By filtering on selected landmarks which formed the sets from previous studies, a direct comparison of this studies' data with previous studies' could be made.

5) Wire-frame construction

Before exporting the processed data to a Morphologika file, a wire-frame is constructed according to the landmarks selected. This enables a simple 3D frame structure to be visualised and manipulated in 3D for each skeletal sample as shown in Figure 14.

Figure 14 shows Os Coxa wireframe from dorsal, cranial and lateral, respectively, perspectives. These show the procrustes mean of 7 species used in this study with 108 point landmarks.

\section{6) Processing in morphologika}

Once in the Morphologika software, the data was processed in the following two steps:

Procrustes Analysis (with reflections enabled) was performed. This process involves a number of steps:

- The centroid (average $x, y, z$ co-ordinate for each morph) is calculated.

- The root mean square (RMS) is calculated for each landmark, giving its distance from the centroid. An average value for the whole morph is calculated.

- The centroid is subtracted from each landmark, translating all morphs to the same centroid around the origin.

- All landmark-centroid distances were divided by the average of the all distances, scaling all morphs to be the same size.

- Finally, each morph is rotated so as to minimise the RMS of the distances of all the landmarks.

Principal Components Analysis. Next a principal components analysis was performed. This process effectively analyses all the 3D data point combinations and selects the ones which provide the greatest variation. These are then labelled principal component $1 \ldots 32$. ( $\mathrm{PC1} \ldots$ PC32.) At the end of the process the morphs are plotted on a chart showing the two largest PCs (1 and 2). Ideally the morphs from the same taxa/sex should cluster together indicating that they have
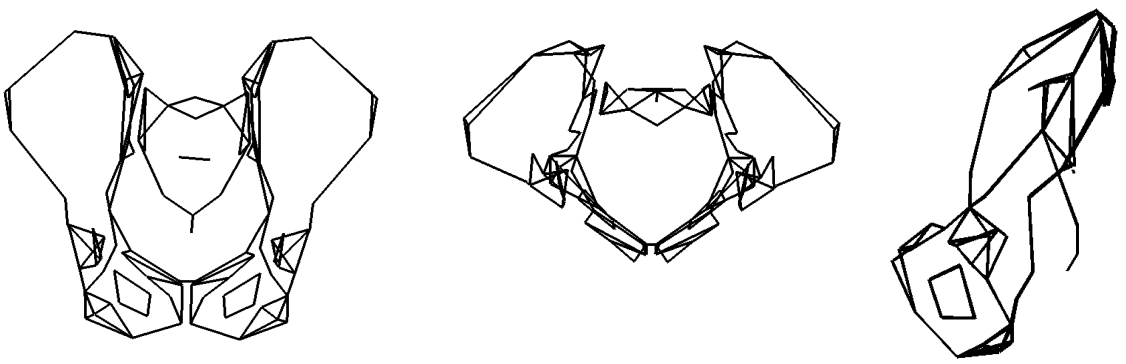

Figure 14. Example wireframes of fully the articulated pelvis. 
similar shape. Differences between clusters on the different PCs can then be analysed to see what might be inferred about them.

\subsection{Relative Muscle Lever Arm Study Method}

Triangular sets of three points were derived for each hip bone landmark associated with a muscle origin for each skeletal sample, including two generated landmarks. The following method was the basis for the calculation of lever arms. It should be noted that it describes a simplified, unspecified muscle. Known caveats and difficulties with the method are described afterwards.

1) A pseudo landmark (landmark 0-AcCenterGen) was generated to represent the centre of rotation of the acetabulum on each hip bone. As shown in Figure 15, it was calculated as the midpoint of four landmarks (2, 3, 4 and 5) on the rim of the acetabulum.

2) Another pseudo landmark was generated to represent a standard position of the greater trochanter of the femur relative to each hip bone. It was calculated, as shown in Figure 16, using a vector from the landmark at the centre of the acetabulum (landmark 1-AcCent) through the calculated landmark just described (landmark 0 AcCenterGen-Figure 16(A)), the centre of rotation of the acetabulum, through to a point away from the hip. The distance was calculated by using the actual length of the femoral head from the same individual (Figure 16(B)). In this way, a landmark on the hip bone was generated to represent a generalised muscle insertion point (Figure 16(C)).

3) On each hip bone, for each landmark representing a muscle origin, the lever arm was calculated using the following procedure:

a) A triangle of $33 \mathrm{D}$ co-ordinates was processed (Figure 17):

$\mathrm{o}=$ the centre of rotation about the centre of the femoral head.

$\mathrm{f}=$ the generated landmark representing the end of the femoral neck.

$\mathrm{m}=$ the landmark representing the muscle origin.

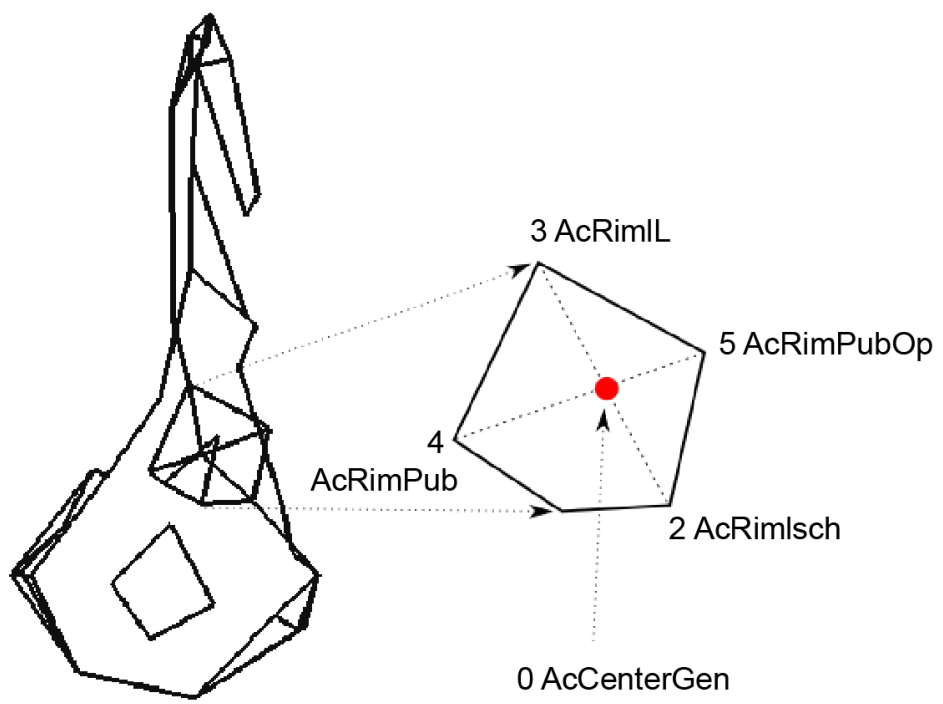

Figure 15. Calculation of pseudo-landmark for centre of rotation of the acetabulum. 


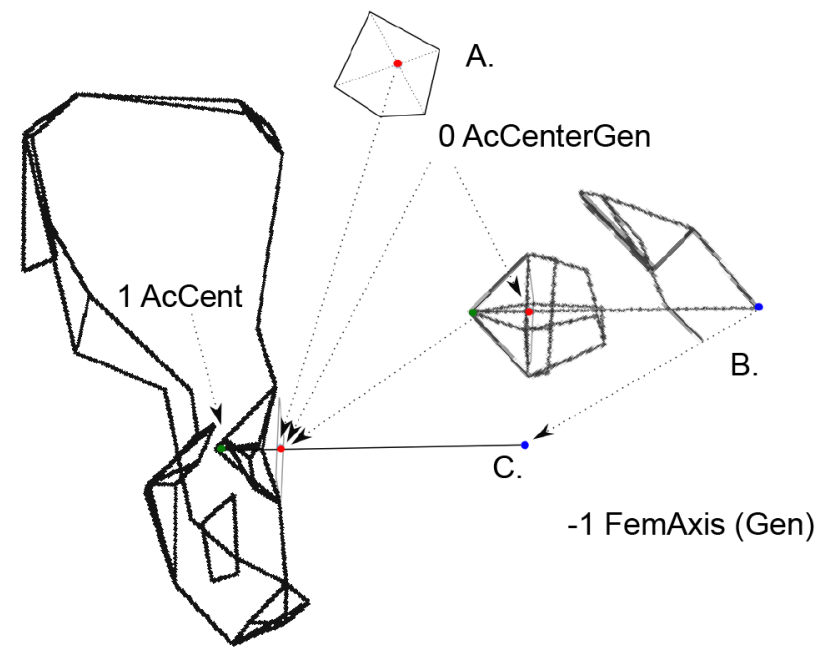

Figure 16. Method of calculation for pseudo landmark (FemAxis).

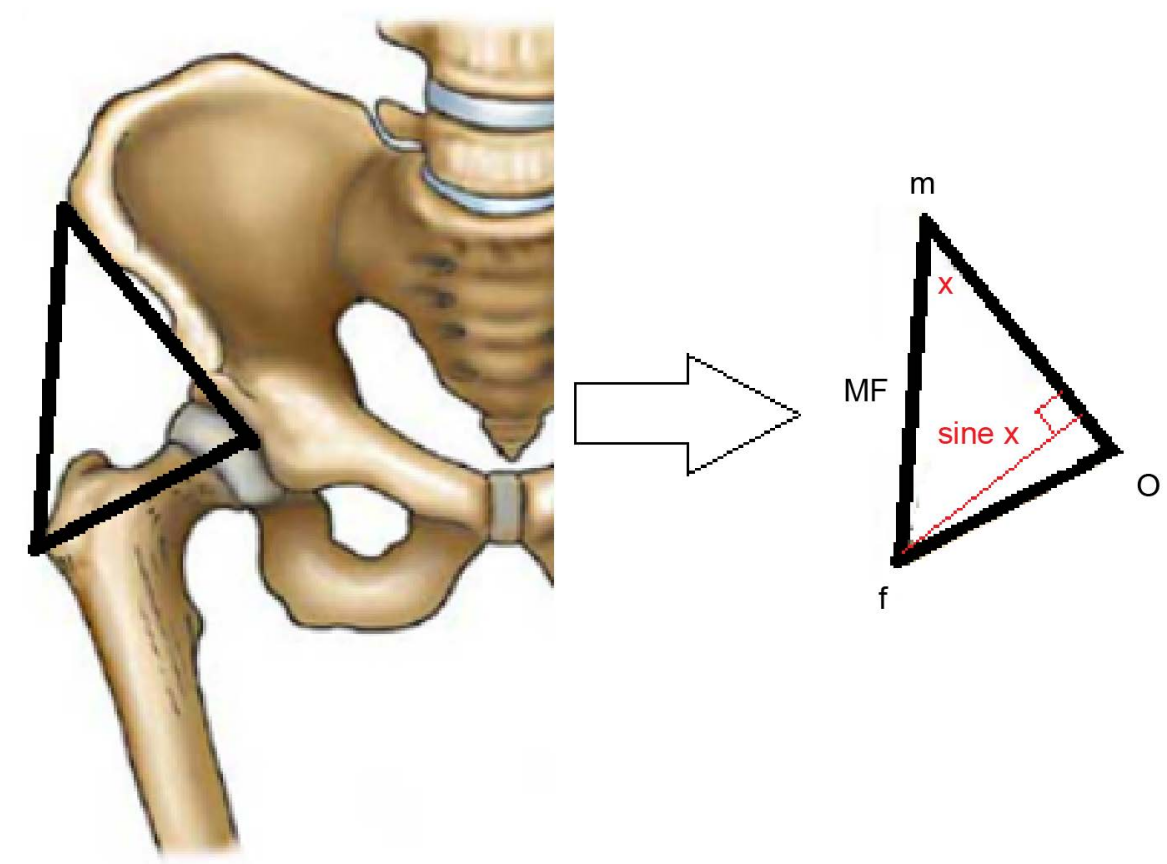

Figure 17. Lever arm calculation.

b) The angle ( $\mathrm{x})$ is calculated for $\langle\mathrm{omf}\rangle$.

c) The distance MF is calculated.

d) MF is multiplied by sine( $\mathrm{x}$ ) to get the lever arm.

\subsubsection{Caveats}

A number of significant limitations of this study should be noted here before describing the results.

1) A single static hip position was assumed

There are thousands of possible permutations of the hip. Obviously, as femur moves relative to the hip bone, any specific muscle lever arm will change greatly. For example, the lever arm of Gluteus medius is very different when the hip is 
extended from when it is flexed. To analyse hip muscle lever arms in full then, one would need not only to consider every muscle lever arm, but to do so in every possible position the hip could be orientated. In order to greatly simplify the study, only one fixed position was assumed. Although this greatly reduces the scope of the study and what might be interpreted from them, it is argued that even this one position will allow the lever arm ratios to be calculated and compared across species, thus elucidating the likely effects on muscle action on the shape of the australopithecine pelvis.

\section{2) All insertion points on the femur were identical}

For similar reasons all muscle insertion points on the femur were estimated to be at the same point-approximating to the most lateral point on the greater trochanter on the femoral axis. As each muscle lever arm greatly depends on the position of the insertion point of the femur relative to the hip, and as the orientation of the femur can vary greatly, it vastly reduces the number of permutations to consider if it is assumed that the femur is in a static position and that all muscles insert at the same point.

Although this is obviously an unrealistic model of the true overall biomechanics of the hip, it will consistently show the shape differences of the hip bone in terms of muscle lever arms against a fixed point in space, one that is the true insertion point for some of the muscles. The generated landmark is calculated using the actual femoral length of the individual in question.

A future study is proposed (see final chapter) to greatly enhance the data set produced to overcome this weakness.

\section{3) Soft tissue and muscle wrapping ignored}

Many muscles, particularly those in the pelvis region, are known to wrap around bone and soft tissue, including other muscle layers. Again for reasons of simplicity these problems were largely ignored in this study. Where muscles are known to wrap around the hip bone significantly (e.g. the superior and inferior gemelli) an estimated muscle origin was estimated to be near the point of wrapping.

Again, it is suggested that despite this limitation the results can still infer the most significant effects of hip bone shape on the locomotion of the hip.

4) Large muscle origins and muscle blocks were grossly simplified

Some hip muscles, such as the gluteal block, originate on relatively large surface areas of the hip bone and can perform more than one muscle action, for example both extension and abduction. For this reason, landmarks were clustered according to likely muscle action, rather than specifically for which muscle origin they were likely to correspond to in the technical sense. Many areas of the hip bone, where large muscles such as these originate, had no corresponding landmark.

5) Paleospecies muscle function is speculation

Although the muscle action of extant species is now well known, the actions of the muscles in the paleospecies used in this study were, of course, based on spec- 
ulation and the principle of homology.

\subsubsection{Morphs Used}

For this study, 70 hip bones were selected for those specimens with corresponding femora from 12 species, including 2 paleospecies: $A$ afarensis and $H$ erectus. (for example $A$. africanus could not be included here because there is no corresponding femoral neck specimen.)

\subsubsection{Processing}

The following procedure was used to derive and analyse the lever arm data:

1) A subset of 44 landmarks of the hip bone were used, from which the lever arm from the centre of the acetabulum to the generated landmark representing the greater trochanter was calculated for muscles of the hip as described above.

2) Each of these calculated lever arms were expressed as a ratio of all the other calculated lever arms for each specimen.

3) The average of each permutation was calculated for each species.

Thus a data set of 1,936 $\left(44^{2}\right)$ lever arm permutations was derived for each specimen, showing the ratio of each lever arm against each other.

One example will be followed through here to demonstrate this process.

\subsubsection{Abduction versus Extension Muscle Block Example}

One key muscle involved in abduction of the thigh is the tensor fasciae lata. The origin of this muscle is identified on the hip bone schema here as landmark 12.

In humans the gluteal muscles also perform this function to a degree. The origin of these muscles is a very large area, however, so to contrast the biomechanical Lever Arm of extension, a landmark was selected that was more central: landmark 16 (Figure 18).

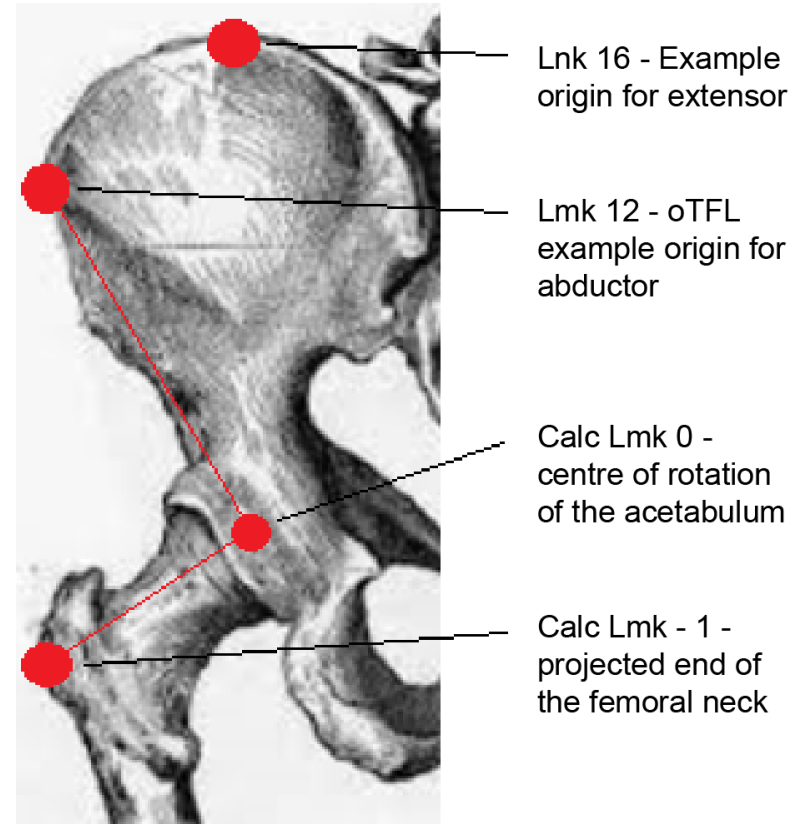

Figure 18. Sample landmarks to compare Abduction v Extension of the Hip. 
1) $3 D$ co-ordinates of these landmarks

Here are a set of sample $3 \mathrm{D}$ co-ordinates for two paleo-species and two randomly selected human specimen (one male, one female).

\begin{tabular}{|c|c|c|c|c|c|}
\hline \multicolumn{6}{|c|}{ AFA002881-HIP-DIG-AK-20050620-461-Australopithecus afarensis } \\
\hline Lmk & $\mathrm{x}$ & $\mathrm{Y}$ & Z & Lever Arm & Ratio $16 / 12$ \\
\hline-1 & 330.51 & -41.3 & 198.41 & & \\
\hline 0 & 316.32 & -66.2 & 178.36 & & \\
\hline 12 & 284.67 & -0.8 & 145.17 & 33.87 & 0.49 \\
\hline 16 & 262.53 & -77.21 & 101.97 & 16.67 & \\
\hline \multicolumn{6}{|c|}{ HER005000-HIP-DIG-AK-20050627-465-Homo erectus } \\
\hline-1 & -273.94 & -146.68 & 209.06 & & \\
\hline 0 & -283.91 & -86.79 & 189.72 & & \\
\hline 12 & -198.13 & -95.15 & 153.76 & 55.24 & 0.78 \\
\hline 16 & -246.96 & -56.83 & 76.69 & 42.71 & \\
\hline \multicolumn{6}{|c|}{ HSSM00029-HIP-DIG-AK-20080520-913-Homo sapienssapiens (Male) } \\
\hline-1 & 179.08 & -295.38 & 287.73 & & \\
\hline 0 & 115.83 & -321.81 & 280.12 & & \\
\hline 12 & 110.74 & -278.48 & 354.05 & 59.51 & 0.67 \\
\hline 16 & 39.22 & -334.65 & 365.76 & 39.78 & \\
\hline \multicolumn{6}{|c|}{ HSSF00054-HIP-DIG-AK-20080813-977-Homo sapienssapiens (Female) } \\
\hline-1 & 250.45 & 1.45 & 421.01 & & \\
\hline 0 & 277.41 & 6.30 & 360.13 & & \\
\hline 12 & 254.10 & -67.89 & 331.31 & 48.34 & 0.67 \\
\hline 16 & 318.29 & -69.71 & 283.31 & 32.49 & \\
\hline
\end{tabular}

Once the load arm ratios have been calculated, they are then averaged in extant species. Here, for just two specimen considered, Homo sapiens would end up with an average ratio of 0.67 .

Finally, all the ratios for all the species are expressed as a ratio of that for $\mathrm{Ho}$ mo sapiens.

So, for this small example...

$\begin{array}{cc}\text { Homo erectus } & 1.16 \\ \text { Homo sapiens } & 1.00 \\ \text { Australopithecus afarensis } & 0.74\end{array}$


This indicates that australopithecines have a smaller lever arm ratio for extension as a percentage of that for abduction of the thigh than the other two species.

\subsubsection{Pivot Table Analysis}

As the data set is relatively large ( $>135,000$ rows) and a degree of flexibility was required in order to perform relevant queries pertaining to the muscle actions of the hip muscles, the data was inserted into a single, large "fact table" which lends itself to the modern business practice of performing a "pivot table" summary analysis on it.

Figure 19 shows one row for each specimen load arm permutation.

Many users of Microsoft Excel will be familiar with this technique and the Excel Workbook containing the Pivot Table is available in the supplementary materials for personal use, if required.

Figure 20 shows the same sample comparison, but for the whole set of hip bones.

Using this method it was possible to group sets of multiple landmarks to represent more widely distributed origins for various muscle blocks and thus generate summary ratio data for all the permutations of the major locomotor groups pertaining to the hip.

\subsubsection{Muscle Action Groupings}

As some muscles are known to perform multiple actions (e.g. Gluteus maximus can act as an extensor, an abductor and a medial rotator) it was decided to simplify the analysis by pooling antagonistic or opposing muscle actions and grouping them into three major categories.

- Flexion/Extension

- Abduction/Adduction

- Lateral/Medial Rotation

The task was then to compare the results from these three major muscle action groups against each other in order to determine the major differences among the species being studied.

\begin{tabular}{|c|c|c|c|c|c|}
\hline$\Delta$ & A & B & $\mathrm{C}$ & $\mathrm{D}$ & $\mathrm{E}$ \\
\hline 1 & MorphCode & $-\dagger$ LatinName & Ratiolmk1 & RatioLmk2 - & Ratio \\
\hline 2 & AFA002881-HIP-DIG-AK-20050620-461 & Australopithecus afarensis & Lmk02 & Lmk02 & 1 \\
\hline 3 & AFA002881-HIP-DIG-AK-20050620-461 & Australopithecus afarensis & Lmk03 & Lmk02 & 1.110351797 \\
\hline 4 & AFA002881-HIP-DIG-AK-20050620-461 & Australopithecus afarensis & Lmk04 & Lmk02 & 1.704344651 \\
\hline 5 & AFA002881-HIP-DIG-AK-20050620-461 & Australopithecus afarensis & Lmk05 & Lmk02 & 1.2551874 \\
\hline 6 & AFA002881-HIP-DIG-AK-20050620-461 & Australopithecus afarensis & Lmk06 & Lmk02 & 1.654133984 \\
\hline 7 & AFA002881-HIP-DIG-AK-20050620-461 & Australopithecus afarensis & Lmk07 & Lmk02 & 1.156905613 \\
\hline 8 & AFA002881-HIP-DIG-AK-20050620-461 & Australopithecus afarensis & Lmk08 & Lmk02 & 2.429374338 \\
\hline 9 & AFA002881-HIP-DIG-AK-20050620-461 & Australopithecus afarensis & Lmk09 & Lmk02 & 0.863955229 \\
\hline 10 & AFA002881-HIP-DIG-AK-20050620-461 & Australopithecus afarensis & Lmk10 & Lmk02 & 0.787900365 \\
\hline 11 & AFA002881-HIP-DIG-AK-20050620-461 & Australonithecus afarensis & Lmk11 & Lmk02 & 0.507185703 \\
\hline
\end{tabular}

Figure 19. Sample of fact table data. 


\begin{tabular}{|c|c|c|c|c|c|}
\hline 4 & \multicolumn{2}{|l|}{ A } & \multicolumn{2}{|c|}{ B } & \multirow[t]{2}{*}{ C } \\
\hline 1 & RatioLmk1 & & Lmk12 & $\mathbf{T}$ & \\
\hline 2 & RatioLmk2 & & Lmk016 & $\mathbf{T}$ & \\
\hline \multicolumn{6}{|l|}{3} \\
\hline 4 & Row Labels & -1 & Average & \multicolumn{2}{|c|}{$\mathrm{N}$} \\
\hline 5 & Lemur catta & & & & 1 \\
\hline 6 & Hylobates moloch & & & & 1 \\
\hline 7 & Cercopithecus erythrotis & & & & 1 \\
\hline 8 & Hylobates muelleri & & & & 1 \\
\hline 9 & Pan troglodytes & & & & 15 \\
\hline 10 & Pongo abelii & & & & 2 \\
\hline 11 & Pan paniscus & & & & 18 \\
\hline 12 & Pongo pygmaeus & & & 15 & 2 \\
\hline 13 & Homo erectus & & & 73 & 1 \\
\hline 14 & Gorilla gorilla graueri & & & 71 & 6 \\
\hline 15 & Homo sapiens sapiens & & & 87 & 21 \\
\hline 16 & Australopithecus afarensis & & & 492 & 1 \\
\hline 17 & Grand Total & & & .749 & 70 \\
\hline
\end{tabular}

Figure 20. Example pivot table.

In order to tease out the differences between these muscle action groups, in terms of this hip bone shape analysis of lever arm ratios, any landmarks that were common to different paired groups were eliminated.

\subsubsection{Hip Muscles Analysed}

The following Table 6 lists the major muscles of the hip concerned with locomotion, their origin and the landmarks used from the schema here to calculate their lever arms.

Where significant muscle wrapping is known, a local "proxy" on the hip bone was used as the landmark from which to calculate the lever arm.

From this, groups of landmarks were derived to represent the three major categories of muscle action: flexion/extension; medial/lateral rotation and abduction/adduction.

Landmarks for Muscle Action Group

$\begin{array}{cc}\text { Flexion/Extension } & 9,11,14,15,17,18,19,20,22,28,29,30,31,38,40,41,42 \\ \text { Rotation } & 17,18,19,20,22,30,31,33,37,38,39,40,42,45 \\ \text { Abduction/Adduction } & 12,14,15,17,18,19,20,22,30,31,32,33,39\end{array}$

Finally, these three groups of landmarks were permuted against each other and common landmarks to each pair were removed.

\begin{tabular}{ccc}
\hline Extension/Flexion (ER) v Rotation & ER: $9,11,14,15,28,29,41$ & R: $33,37,39,45$ \\
Extension/Flexion v Abduction/Adduction & ER: $9,11,28,29,38,40,41,42$ & A: $12,32,33,39$ \\
Rotation v Abduction/Adduction & R: $37,38,40,42,45$ & A: $12,14,15,32$ \\
\hline
\end{tabular}


Table 6. Landmarks used in muscle load arm calculations.

\begin{tabular}{|c|c|c|}
\hline Muscle & Origin as used on Hip bone (or proxy) & Landmarks \\
\hline \multicolumn{3}{|l|}{ Flexors } \\
\hline Iliacus & Illiac fossa (Proxy used on pelvic brim) & 28,29 \\
\hline Psoas & lumbar vertebrae (Proxy used on pelvic brim) & 28,29 \\
\hline Pectineus & Pectineal line on superior ramus of pubis & 30 \\
\hline Adductus longus & Pubic tubercle & 31 \\
\hline Rectus femoris & Anterior Superior Iliac Spine (ASIS) & 9,11 \\
\hline Sartorius & ASIS & 11 \\
\hline \multicolumn{3}{|l|}{ Extensors } \\
\hline Semitendinosus & Ischial tuberocity & 40,42 \\
\hline Biceps femoris & Ischial tuberocity & 40,42 \\
\hline Semimembranosus & Ischial tuberocity & 38,41 \\
\hline Gluteus maximus & Medialedge of ilium & $17,18,19,20$ \\
\hline Gluteus medius & Gluteal surface of ilium & $15,22,14$ \\
\hline Gluteus minimus & Gluteal surface of ilium & $14,12,22$ \\
\hline \multicolumn{3}{|l|}{ Medial rotators } \\
\hline Pectineus & Pectineal line on superior ramus of pubis & 30 \\
\hline Adductus longus & Pubic Tubercle & 31 \\
\hline Adductus magnus & Inferior ramus of pubis\& Inferior ramus of ischium & 39,33 \\
\hline Obturator internus & Inner surface of Obturator foramen (sciatic notch) & 37 \\
\hline \multicolumn{3}{|l|}{ Lateral rotators } \\
\hline Gemelllus superior & Ischial spine & 37 \\
\hline Gemelllus inferior & Above Ischial tuberocity & 37 \\
\hline obturator externus & Medial margin of obturator foramen & 45 \\
\hline Piriformis & Closest point on sciatic notch to the sacrum & 22 \\
\hline Quadradus femoris & Exterior border of the ischial tuberocity & 38,40 \\
\hline Biceps femoris & Ischial tuberocity & 40,42 \\
\hline gluteus maximus & Gluteal surface of ilium & $17,18,19,20$ \\
\hline \multicolumn{3}{|l|}{ Abductors } \\
\hline TFL & ASIS & 12 \\
\hline Gluteus medius & Gluteal surface of ilium & $15,22,14$ \\
\hline Gluteus maximus & Gluteal surface of ilium & $17,18,19,20$ \\
\hline \multicolumn{3}{|l|}{ Adductors } \\
\hline Adductor longus & Pubic tubercle & 31 \\
\hline adductor brevis & Pubic tubercle and inferior towards ramus & 31,32 \\
\hline Adductor magnus & Along inferior ramus of ischium and pubis & 39,33 \\
\hline Gracilis & Lower pubic symphysis to upper half of public arch & 33 \\
\hline Pectineus & Pectineal line on superior ramus & 30 \\
\hline
\end{tabular}


These sets of landmarks were then used as filters in the pivot table analysis to generate average lever arm ratios for all the permutations of landmark pairs. 